Please quote as: Kummer, T.-F.; Leimeister, J. M. \& Bick, M. (2012): Die Bedeutung von nationaler Kultur für die Gestaltung von Informationssystemen. In: WIRTSCHAFTSINFORMATIK, Ausgabe/Number: 6, Vol. 54, Erscheinungsjahr/Year: 2012. Seiten/Pages: 303-318. 


\section{Die Bedeutung von nationaler Kultur für die Gestaltung von Informationssystemen}

Kultur ist in Form von geteilten Werten und Verhaltensweisen für die Entwicklung von soziotechnischen Systemen von zentraler Bedeutung. Aufgrund der damit verbundenen Vielfalt an Problem- und Fragestellungen sowie der Betrachtung unterschiedlicher Kulturformen weisen bisherige Veröffentlichungen eine erhebliche Heterogenität auf. Im vorliegenden Beitrag wird daher ein Ordnungsrahmen zur kultursensitiven Gestaltung von Informationssystemen erarbeitet, der eine Strukturierung bisheriger Arbeiten ermöglicht. Darauf aufbauend wird anhand einer Literaturanalyse untersucht, welche Berührungspunkte zwischen Kultur und den Elementen der Systementwicklung bisher bearbeitet wurden. Somit wird gezielt herausgearbeitet, welche Erkenntnisse zu den verschiedenen Themenbereichen der kultursensitiven Gestaltung von Informationssystemen in der Literatur vorliegen und wo zukünftige Forschungsbedarfe auf dem Weg hin zu entsprechendem Gestaltungswissen liegen.

DOI 10.1007/s11576-012-0340-4

\section{Die Autoren \\ Dr. Tyge-F. Kummer ( $₫)$ \\ Institut für Wirtschaftsinformatik Wirtschaftswissenschaftliche Fakultät Humboldt-Universität zu Berlin Spandauer Straße 1 \\ 10178 Berlin \\ Deutschland \\ tyge.kummer@hu-berlin.de}

Prof. Dr. Jan Marco Leimeister Institut für Wirtschaftsinformatik Universität St. Gallen

Mueller-Friedberg-Strasse 8

9000 St. Gallen

Schweiz

JanMarco.Leimeister@unisg.ch und

Fachgebiet Wirtschaftsinformatik

Universität Kassel

Pfannkuchstr. 1

34121 Kassel

Deutschland

leimeister@uni-kassel.de

Prof. Dr. Markus Bick

Lehrstuhl für Wirtschaftsinformatik ESCP Europe Wirtschaftshochschule Berlin

Heubnerweg 8-10

14059 Berlin

Deutschland

mbick@escpeurope.de
Eingegangen: 2011-11-22

Angenommen: 2012-09-06

Angenommen nach zwei Überarbeitungen durch Prof. Dr. Armin Heinzl.

Online publiziert: 2012-11-08

This article is also available in English via http://www.springerlink.com and http://www.bise-journal.org: Kummer T-F, Leimeister JM, Bick $M$, (2012) On the Importance of National Culture for the Design of Information Systems. Bus Inf Syst Eng. doi: 10.1007/s12599-012-0236-2.

Zusätzliche Information ist in der Online-Version dieses Beitrags (doi: 10.1007/s11576-012-0340-4) enthalten.

(c) Springer Fachmedien Wiesbaden 2012

\section{Einleitung}

Nationale Kultur - im Sinne von gemeinsamen Werten und Verhaltensnormen der Individuen in einem Land (Straub et al. 2002) - beeinflusst die Gestaltung von Informationssystemen maßgeblich (Leidner und Kayworth 2006). Als Beispiele lassen sich unter anderem der Erfolg von unterschiedlich gestalteten Web- seiten, mobilen Geräten oder mobilen Diensten in der westlichen Welt und Asien anführen (Ishii 2004; Choi et al. 2005). Diese Unterschiede sind für die Gestaltung von Informationssystemen von zentraler Bedeutung, um in den jeweiligen Kulturkreisen die gewünschten Funktionalitäten bereitzustellen, die Nutzung der Systeme zu vereinfachen und Akzeptanzprobleme zu vermeiden (Ishii 2004; Choi et al. 2005; Leidner und Kayworth 2006). Darüber hinaus können unterschiedliche Wertevorstellungen seitens der Systemgestalter gerade bei internationalen Entwicklungsprojekten zu Problemen führen, die den Einführungserfolg gefährden (Winkler et al. 2007; Keil et al. 2007; Tan et al. 2003; Yuan und Vogel 2006).

Bereits seit den 1970er-Jahren wird der Einfluss von Kultur auf Informationssysteme untersucht (Gallivan und Srite 2005). Leidner und Kayworth (2006) identifizieren insgesamt 85 Forschungsarbeiten, die den Einfluss von nationaler und organisationaler Kultur auf Informationssysteme behandeln. Diese lassen sich sechs Themengebieten zuordnen (Culture and IS Development; Culture, IT Adoption and Diffusion; Culture, IT Use and Outcomes; Culture, IT Management and Strategy; IT's Influence on Culture; IT Culture). Dabei lassen sich zehn Beiträge dem gerade aus Sicht einer gestaltungsorientierten Wirtschaftsinformatik besonders relevant anzusehenden Themengebiet „Culture and 
Abb. 1 Modelle zur Konzeptualisierung von Kultur: Schein (2004) als Beispiel für Organisationskultur, Karahanna et al. (2005) als Beispiel für unterschiedliche Kulturschichten und deren Struktur

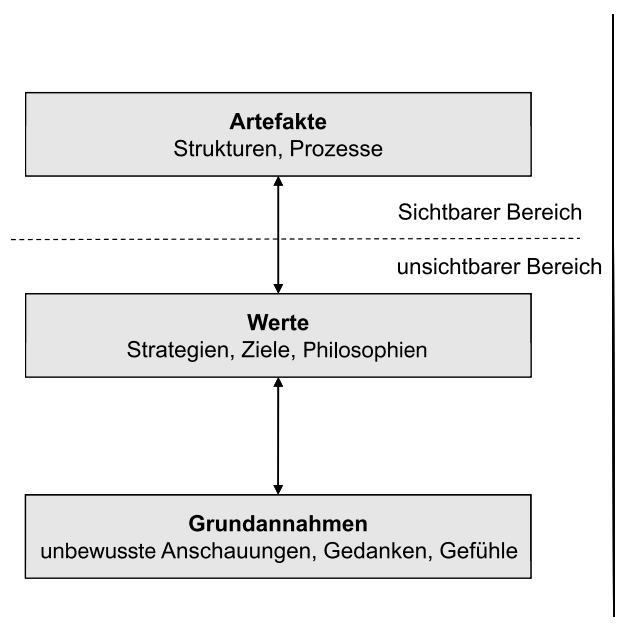

Ebenenmodell nach Schein (2004)

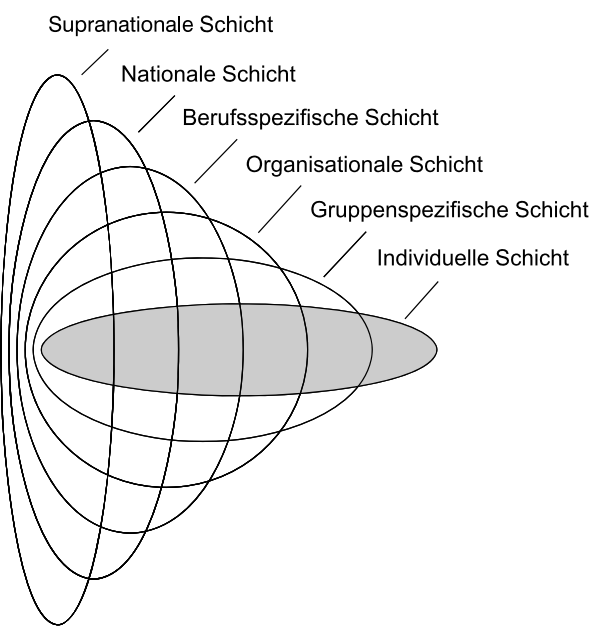

Virtuelles Zwiebelmodell nach Karahanna et al. (2005)
IS Development“ zuweisen. Doch auch dem Bereich „IT Adoption and Diffusion" können Beiträge zugeordnet werden, die bei der Gestaltung von Informationssystemen aus kultureller Sicht beachtet werden sollten. Von besonderer Relevanz erscheint hierbei insbesondere, kulturelle Anforderungen an Informationssysteme in Zielmärkten zu adressieren, denn das Beachten kultureller Anforderungen an Systeme und Produkte können deren Erfolg maßgeblich beeinflussen (Marcus und Gould 2000). Die übergeordnete Ziel des vorliegenden Beitrags ist, den Wissenstand zur Rolle von Kultur für das Design von Informationssystemen aufzuzeigen. Aufgrund der hohen Heterogenität der Forschungsarbeiten in diesem Themengebiet bedarf es einer dezidierten Literaturanalyse, um systematisch herauszuarbeiten, welche Ergebnisse vorliegen und welche Forschungsfragen bisher unbeantwortet sind.

In diesem Beitrag werden daher in einem ersten Schritt bereits existierenden Forschungsergebnisse zur Bedeutung der nationalen Kultur für die Gestaltung von Informationssystemen entlang eines dezidierten Ordnungsrahmens strukturiert und verdichtet. Darauf aufbauend werden dann in einem weiteren Schritt über die reine Ordnung hinaus - die mit den Themen in Beziehung stehenden Forschungslücken herausgearbeitet. Dabei werden die folgenden Fragestellungen beantwortet:

1. Welche Forschungsergebnisse wurden erzielt und welche Forschungsmethoden wurden dabei verwendet?

2. Welche Forschungspotenziale bestehen und wie können diese erschlossen werden?
3. Welche theoretischen Modelle werden eingesetzt, um die nationale Kultur mit der Gestaltung von Informationsund Kommunikationssystemen zu verbinden?

Im Folgenden wird zunächst der der Literaturanalyse zugrundeliegende Ordnungsrahmen erläutert (Abschn. 2), bevor darauf aufbauend die konkrete Analysearbeit beschrieben wird (Abschn. 3). In Abschn. 4 werden dann die korrespondierenden Ergebnisse der Untersuchung vorgestellt und deren Implikationen im darauf folgenden Abschn. 5 erläutert. Der Beitrag endet mit einem Fazit hinsichtlich der erzielten Ergebnisse und deren Nutzen für die Wirtschaftsinformatik.

\section{Entwicklung eines Ordnungsrahmens}

Das zentrale Erkenntnisobjekt der Wirtschaftsinformatik - die Informationssysteme - bildet die Grundlage des Ordnungsrahmens (Abschn. 2.1), entlang dessen die während der Literaturrecherche identifizierten Arbeiten klassifiziert werden. Darüber hinaus gilt es, das diesem Beitrag zugrundeliegende Kulturverständnis darzulegen (Abschn. 2.2). Abschließend wird dann der Ordnungsrahmen entwickelt, der die Gestaltung von Informationssystemen mit Kultur verbindet.

\subsection{Gestaltung von Informationssystemen}

Die Gestaltungsorientierung definiert eine zentrale Strömung insbesondere in- nerhalb der deutschsprachigen Wirtschaftsinformatik (Fettke et al. 2010). Ziel ist es, mittels innovativer Techniken - beispielsweise in Form von Methoden, Modellen und Softwareprototypen - Artefakte zu entwickeln, die zur Lösung von praktischen Problemen eingesetzt werden können (Becker 1995; Hevner et al. 2004). Dabei erfordert die Systemgestaltung ein Gestaltungssubjekt, welches die Gestaltung vornimmt. Hierbei handelt es sich im Allgemeinen um Menschen (z. B. Business Analyst), die mittels einer Gestaltungstechnik (z. B. Unified Modeling Language - UML) ein Gestaltungsziel (z. B. Abbildung von Geschäftsprozessen) verfolgen. Demgegenüber handelt es sich bei dem Gestaltungsobjekt um soziotechnische Systeme. Dem Ansatz von Sydow (1988) folgend umfasst das Gestaltungsobjekt eine strukturierte Menge von Aufgaben (z. B. Geschäftsprozesse) und Technologien (z. B. Business-ProcessManagement-System), die das technische Subsystem darstellen, sowie Mitgliedern (z. B. Anwendern) und Rollen (z. B. KeyAccount-Management), die das soziale Subsystem umfassen. Die menschlichen und maschinellen Komponenten (Teilsysteme) dienen dabei (Heinrich et al. 2011; o. V. 2011). In Abb. 1 werden diese Zusammenhänge sowie die Beziehung zwischen Gestaltungssubjekt und Gestaltungsobjekt schematisch dargestellt.

Die Gestaltung von Informationssystemen wird aus Gründen der Komplexitätsreduktion und dabei zur Strukturierung des Entwicklungsprozesses aber auch zur Unterstützung des entsprechenden Projektmanagements mithilfe von Vorgehensmodellen in einzelne Phasen bzw. Arbeitsschritte unterteilt (z. B. 
Stahlknecht und Hasenkamp 2004). Gerade die mit der Gestaltung von Informationssystemen verbundenen typischen Projektphasen sind bei der Entwicklung des Ordnungsrahmens und damit bei der Literaturanalyse zu berücksichtigen.

\subsection{Kultur und Kulturdimensionen}

In der Kulturforschung werden Differenzen und Gemeinsamkeiten von Menschen mit unterschiedlichem kulturellem Hintergrund analysiert, um ein besseres Verständnis der mit Kultur verbundenen Wirkungsprozesse zu gewinnen (Straub et al. 2002). Dabei wird Kultur zwar umfassend thematisiert, eine eindeutige und vor allem allgemeingültige Definition besteht jedoch nicht. So identifizieren Kroeber und Kluckhohn (1952, S. 77 ff.) bereits 162 unterschiedliche Definitionen von Kultur. Dabei handelt es sich um ein komplexes und schwer greifbares Phänomen, welches sowohl Orientierungsmuster als auch die damit verbundenen Vermittlungsmechanismen und Ausdrucksformen umfasst (Schreyögg 1999). In diesem Zusammenhang stellen geteilte Normen und gemeinsame Werte in zahlreichen Definitionen ein zentrales Merkmal dar (Straub et al. 2002). Dieses Verständnis von Kultur wird auch dem vorliegenden Beitrag zugrunde gelegt.

Grundsätzlich werden in der Kulturforschung verschiedene Ansätze vertreten, die sich nicht nur auf unterschiedliche Definitionen, sondern auch unterschiedliche Bezugsobjekte bzw. Schichten und Anwendungsbereiche beziehen. Im Folgenden fassen wir diese Ansätze zu drei übergeordneten Arten von Kulturforschung zusammen: national, organisational und Gruppe. Fokus dieses Beitrags ist die nationale Kultur. Denn sowohl beim Gestaltungssubjekt als auch beim sozialen Subsystem auf Gestaltungsobjektebene handelt es sich üblicherweise um Menschen die vornehmlich durch ihr jeweiliges nationales Umfeld geprägt werden. Dementsprechend wird im Folgenden zunächst nationale Kultur von den beiden anderen Arten abgegrenzt bzw. werden die jeweiligen Wechselwirkungen zwischen den einzelnen Kulturarten aufgezeigt. Ziel ist es, eine angebrachte Abgrenzung zu erarbeiten, nicht zuletzt um im weiteren Verlauf des Beitrags Querbezüge aufzeigen zu können. Dabei reflektieren wir die drei Arten vornehmlich aus Sicht der Kulturforschung. Bezüge zur Informationssys- temgestaltung werden dann im weiteren Verlauf des Beitrags herausgearbeitet.

\subsubsection{Nationale Kulturforschung}

In der nationalen Kulturforschung werden Einstellungs- und Verhaltensunterschiede zwischen Menschen aus unterschiedlichen Ländern untersucht. Der bis heute am weitesten verbreitete Beitrag in diesem Bereich stammt von Hofstede (1980). Auf Basis der per Fragebogen bei IBM erhobenen Daten identifizierte Hofstede vier Kulturdimensionen (Machtdistanz, Individualismus versus Kollektivismus, Maskulinität versus Femininität und Unsicherheitsvermeidung), die die Mitarbeiter in den verschiedenen Ländern voneinander unterscheidet. Später ergänzte Hofstede diese Dimensionen um die Zeitorientierung (Hofstede und Bond 1998). Kritisiert wird an Hofstedes Arbeit insbesondere das Alter der genutzten Daten sowie die ausschließliche Berücksichtigung von IBM-Mitarbeitern, die eine Verzerrung durch die Organisationskultur bedingen kann (Erez und Earley 1993, S. 55; House et al. 1997). Dennoch werden Hofstedes Arbeiten im Umfeld der Wirtschaftsinformatik nach wie vor häufig zitiert (Gallivan und Srite 2005; Leidner und Kayworth 2006). Neben Hofstede haben auch andere Autoren Kulturdimensionen identifiziert. So identifizieren Hall und Hall (1990) mit den Dimensionen Raumverständnis, Kontextbezug, Zeitverständnis und Geschwindigkeit von Nachrichten vier alternative Dimensionen. Weitere Konstrukte werden von Schwartz (1992) und Hampden-Turner und Trompenaars (1993) vorgestellt. Darüber hinaus wurde im Rahmen der Global-Leadership-andOrganizational-Behaviour-Effectiveness(GLOBE-)Studie bewusst versucht, die Schwächen von Hofstedes Ansatz zu kompensieren (House und Javidan 2004). Die Ergebnisse erweitern bzw. konkretisieren Hofstedes Kulturdimensionen, sodass insgesamt neun Dimensionen abgeleitet werden (Durchsetzungsfähigkeit, geschlechtliche Gleichberechtigung, Gruppenkollektivismus, Humanorientierung, institutioneller Kollektivismus, Leistungsorientierung, Machtdistanz, Unsicherheitsvermeidung und Zukunftsorientierung). Darüber hinaus wird - im Gegensatz zu Hofstede - zwischen Praktiken (dem Ist-Zustand) und Werten (dem Soll-Zustand) jeder
Dimension unterschieden, um eine Trennung zwischen gelebten und angestrebten Kulturwerten in einer Nation zu erreichen.

Daneben gibt es auch Kritik am Konzept der nationalen Kultur, so merken z. B. Myers und Tan (2002) an, dass Nationen grundsätzlich ein Phänomen der jüngeren Geschichte sind. Effektive und mit entsprechender Macht ausgestattete nationale Regierungen bildeten sich in Europa und den USA bspw. erst im 19. Jahrhundert. Daher ist es problematisch Nationen mit Kulturen gleichzusetzen, die gegebenenfalls schon seit mehreren tausend Jahren bestehen. Weiterhin verändern sich nationale Staaten kontinuierlich. Hier ist unter anderem an die Auflösung der Union der Sozialistischen Sowjetrepubliken (UdSSR) oder Jugoslawiens zu denken. Zudem ist die zugrundeliegende Idee, jede Nation würde einer Kultur entsprechen, unzutreffend. So wurde Indien ohne gemeinsame Sprache und Ethnie gegründet und weist eine Vielzahl verschiedener Kulturen auf. In Afrika wurden viele Nationen durch die vorherrschenden Kolonialmächte gegründet ohne Berücksichtigung von kulturellen und ethnischen Gesichtspunkten (Myers und Tan 2002). Vor diesem Hintergrund erscheint es realistischer davon auszugehen, dass eine Nation mehr als eine Kultur oder auch mehrere Subkulturen beinhaltet. (Huo und Randall 1991; Peppas 2001; Martinsons und Ma 2009). Die im Folgenden beschriebenen Ansätze wählen ein weiteres Bezugsobjekt (Organisation oder Gruppe), wodurch sie diesem Kritikpunkt entgehen.

\subsubsection{Organisationale Kulturforschung}

Hier stehen die gemeinsame Werte und Verhaltensmuster von Angehörigen jeweils einer Organisation im Vordergrund. Nach Hofstede et al. (2010) handelt es sich hierbei um ein grundsätzlich anderes Kulturphänomen als bei der nationalen Kultur, weshalb diese Arten strikt getrennt werden sollten. Einer der bekanntesten Erklärungsansätze organisationaler Kultur ist das Ebenenmodell von Schein (2004). Diesem Ansatz (Abb. 1 - links) folgend lässt sich Unternehmenskultur in drei Ebenen unterteilen. Auf der obersten Ebene befinden sich die sichtbaren Artefakte (z. B. verwendete Sprache, Kleidung und Technologie). Darunter befindet sich die nicht direkt sichtbare Ebene der Werte. Gemeinsame Wertevorstellungen reduzieren die Unsicherheit 
der Organisationsmitglieder beim Treffen von Entscheidungen in neuen Situationen und dienen daher als Orientierungsrahmen. Auf der untersten Ebene befinden sich die sog. Grundannahmen. Werte, die über einen langen Zeitraum erfolgreich sind, können sich zu Grundannahmen weiterentwickeln. Es besteht somit ein wesentlicher Unterschied gegenüber den Werten in Bezug auf das Ausmaß an Verankerung innerhalb der Organisation. Bei den Grundannahmen ist diese Verankerung so stark, dass eine kritische Diskussion nicht mehr möglich ist und Organisationsmitglieder, die sich nicht an die Grundannahmen halten, aus der Organisationsgemeinschaft ausgeschlossen werden (Schein 2004).

\subsubsection{Gruppenkulturforschung}

Neben den beiden vorherigen Ansätzen lassen sich in den vergangenen Jahren zahlreiche Publikationen identifizieren, die weder der nationalen noch der organisationalen Kultur dezidiert zuzuordnen sind (siehe bspw. Martinsons und Ma 2009; Sarker und Sarker 2009 sowie Rai et al. 2009): Diese Arbeiten thematisieren vornehmlich Gruppenkulturen, die bspw. in Communities auftreten. Eine theoretische Erklärung für derartige Kulturformen liefert $u$. a. die Theorie der sozialen Identifikation, die auf dem Selbst-Konzept basiert (Tajfel 1972; Tajfel und Turner 1979). Diesem Ansatz folgend ergibt sich das Selbst eines Individuums aus Gruppenzugehörigkeiten (Nationalität, Beruf und Organisation) sowie dem konkreten Kontext. Statt einer starren Persönlichkeit wird davon ausgegangen, dass zahlreiche Persönlichkeitsfacetten bestehen, die in verschiedenen Situationen das Verhalten determinieren (Tajfel 1972; Tajfel und Turner 1979). Karahanna et al. (2005) entwickeln aufbauend auf der Theorie das sogenannte „virtuelle Zwiebelmodell“ (Abb. 1 - rechts). Dabei ordnen sich um den individuellen Kern der Persönlichkeit verschiedene Gruppenzugehörigkeiten wie Zwiebelschalen an, die dynamisch die jeweilig dominante Kultur bilden. Da die Anzahl der berücksichtigten Gruppenzugehörigkeiten unbegrenzt ist, kann der Ansatz äußerst flexibel genutzt werden, um unterschiedliche Gruppenkulturen zu untersuchen.

\subsection{Konzeption des Ordnungsrahmens}

Der Ordnungsrahmens baut auf dem Modell der Systemgestaltung nach Fett- ke et al. (2010) auf, welches als Synthese zahlreicher entsprechender Konzepte eine umfassende, aggregierte Basis bildet (Abschn. 2.1). Dabei wurde dieses Modell in Anlehnung an die zuvor getroffene Abgrenzung des Gestaltungsobjekts - als soziotechnisches System auf dieser Ebene um den Ansatz von Sydow (1988) detailliert (Abb. 2). Danach wurden die einzelnen Komponenten bzw. Ebenen dieses erweiterten Modells in die entsprechenden Kulturarten (Abschn. 2.2) eingebettet. Wir folgen der Annahme, dass sowohl Gestaltungssubjekt (z. B. Business Analyst in einem bestimmten Land) als auch Gestaltungsobjekt (z. B. Einführung eines BusinessProcess-Management-Systems in einem weiteren Land) grundsätzlich durch die jeweilige nationale Kultur geprägt werden, in der sie verankert sind (Abschn. 2.2), sodass die Gestaltung eines Informationssystems von dieser Kulturart dominiert wird.

Bei der Einbettung in die organisationale Kultur folgen wir Schein (2004), demzufolge Kultur neben einem sichtbaren Bereich auch nicht direkt zugängliche Komponenten umfasst (Abschn. 2.2). Diese sind mit den beteiligten Akteuren - Gestaltungssubjekt (z. B. Business Analyst) und Gestaltungsobjekt (z. B. KeyAccount-Manager der CRM-System zu Analysezwecken verwendet) - verbunden und werden von diesen als selbstverständlich wahrgenommen. Nicht direkt zugänglichen Kulturkomponenten stellen Werte und Grundannahmen dar, deren Diversität oftmals mit Risiken für die Gestaltung von Informationssystemen in Verbindung gebracht wird. So können die unterschiedlichen Werte auch zu anderen Funktionalitätsanforderungen führen (Ishii 2004; Choi et al. 2005) deren Nichtbeachtung die Effizienz und Effektivität des Systems mindert.

Der in Abschn. 2.2 beschriebene Ansatz des virtuellen Zwiebelmodells (Karahanna et al. 2005) unterstreicht unseres Erachtens, dass nationale Kultur nur eine von vielen Kulturschichten ist, die sich wiederum gegenseitig überlappen und somit stark miteinander verzahnt sind. So beeinflusst letztendlich die jeweilige nationale Kultur (z. B. französisch/deutsch) auch die organisationale Kultur (z. B. zentralisiert/dezentralisiert) oder die Gruppenkultur (z. B. spezialisiert/umsatzorientiert). Dementsprechend bleibt auch die Bedeutung weiterer Kulturarten für die Gestaltung von Informationssystemen grundsätzlich $\mathrm{zu}$ betrachten, um ein vollständiges Bild in diesem Bereich zu entwickeln.

Aufgrund der Zielsetzung des vorliegenden Beitrags sowie der zuvor erläuterten Fokussierung auf die nationale Kultur, rücken organisationale und gruppenspezifische Aspekte in den Hintergrund. Dies wird innerhalb des Ordnungsrahmens bzw. Abbildung 2 durch eine Anpassung der Schriftfarbe (schattiert) herausgestellt.

Der hier beschriebene Ordnungsrahmen (Abb. 2) gibt erste generelle Hinweise zur gezielten - kulturspezifischen - Gestaltung von Informationssystemen und zeigt dabei entsprechende Schnittstellen bzw. Forschungspotenziale auf. Er kann aber auch als Grundlage zur Beantwortung der eingangs angeführten Fragestellungen genutzt werden, in dem die innerhalb der Literaturrecherche identifizierten Arbeiten einerseits dem Gestaltungssubjekt oder -objekt bzw. deren Verbindung zugeordnet werden. Andererseits kann die jeweils im Vordergrund stehende Kulturart gefasst werden; im Folgenden vornehmlich nationale Kultur (Tab. 1). Zur weiteren Strukturierung der einzelnen Ergebnisse können diese wiederum typischen Phasen der Gestaltung von Informationssystemen zugeordnet werden (Abschn. 2.1). Die konkrete Anwendung des Ordnungsrahmens zur strukturierte Analyse bestehender Forschung wird im folgenden Abschnitt näher erläutert.

\section{Methodik}

Die eingesetzte Methodik zur Identifikation geeigneter Beiträge orientiert sich sowohl an Buhl et al. (2011) als auch Piccoli und Ives (2005). Die Vorgehensweise basiert auf einer Stichprobe publizierter Forschungsbeiträge in Journals und Tagungsbänden (siehe ausführlich in Online-Anhang B). Mittels einer systematischen Datenbanksuche wurden dafür anhand von Suchbegriffen Beiträge identifiziert, die sich mit dem Thema Kultur in der Informationssystementwicklung beschäftigen oder in einem direkten Zusammenhang zu diesem Thema stehen. Da ein Überblick über den aktuellen Stand der Forschung gegeben wird, wurden nur Forschungsbeiträge berücksichtigt, die zwischen Januar 2000 und Juli 2011 erschienen sind. Eine ausführliche Erläuterung der gewählten Datenbanken, der verwendeten Suchbegriffe sowie der identifizierten Beiträge im Rahmen der mehrstufigen Konsolidierung ist 
Abb. 2 Ordnungsrahmen zur Literaturanalyse (eigene Darstellung)

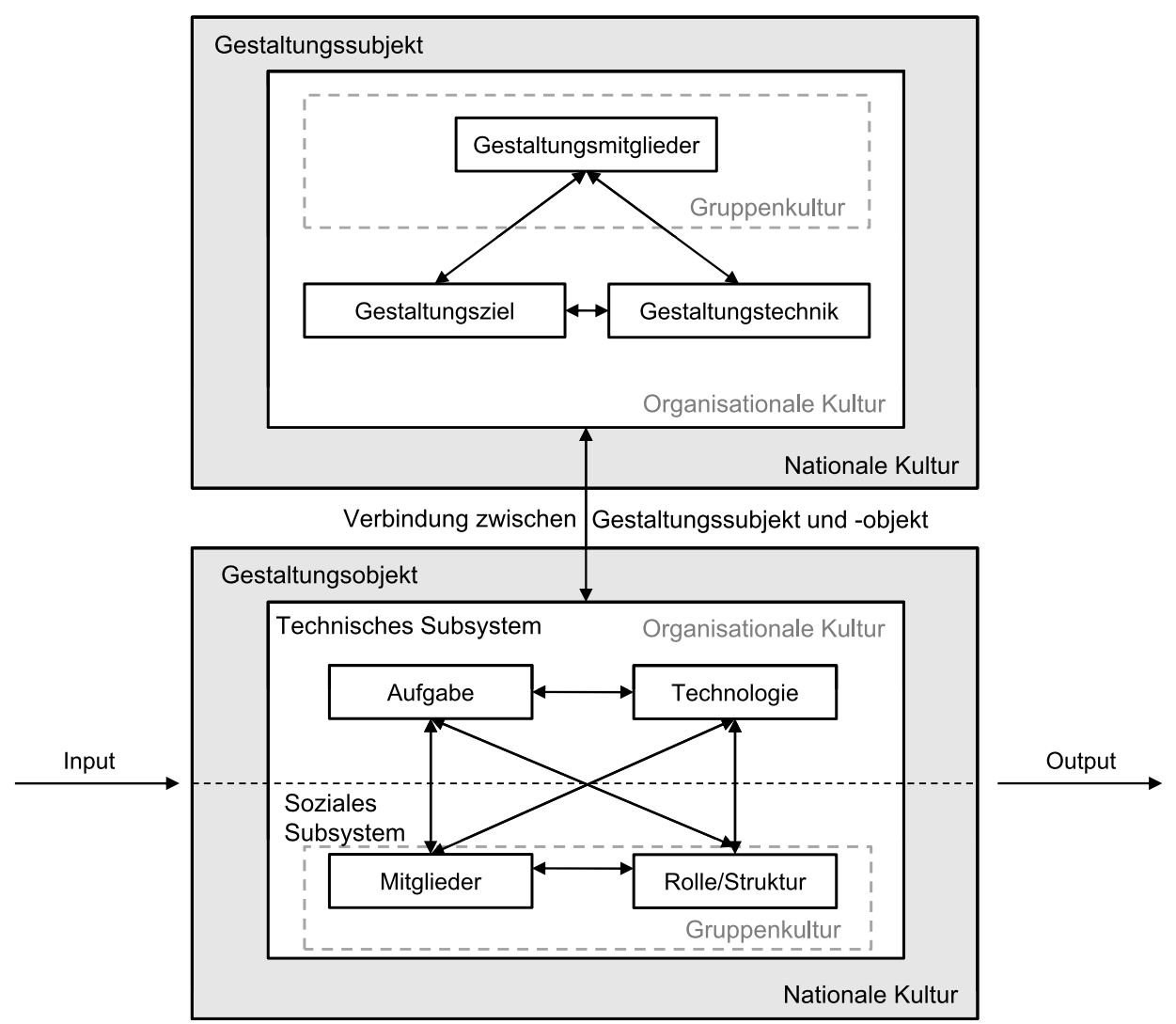

in Online-Anhang B enthalten. Insgesamt wurden 39 Beiträge aus 12 Journals und 7 Tagungsbänden identifiziert.

Bezüglich der Strukturierung und Darstellung der Ergebnisse im Sinne einer Konzeptfokussierung und der Erläuterung der Ergebnisse (Sprachstil und Zeitform) wurde Webster und Watson (2002) gefolgt. Dabei wurden die Beiträge im Sinne des Ordnungsrahmens (Abb. 2) zunächst den Ebenen des Modells der Systemgestaltung zugeordnet. Hierbei wurde zwischen den Ebenen des Gestaltungssubjekts, des Gestaltungsobjekts und der Verbindung zwischen Gestaltungssubjekt und -objekt unterschieden. Anschließend wurden die identifizierten Themen zu Schwerpunkten verdichtet und typischen Phasen der Systementwicklung zugeordnet (Abschn. 2.1). Dabei wird zwischen Vorphase, Analysephase, Entwurfsphase, Realisierungsphase und Einführungsphase nach Stahlknecht und Hasenkamp (2004) unterschieden. Ergänzend wurde eine phasenübergreifende Kategorie zugefügt. Sofern inhaltliche Aspekte eine weitere Gliederung innerhalb einer Phase ermöglichten, wurden die Beiträge in mehrere Themenbereiche untergliedert (Tab. 1). Da bspw. die Entwurfsphase auch die Auswahl und Nutzung einer angemessenen Entwick- lungsmethode voraussetzt (Stahlknecht und Hasenkamp 2004), werden in dieser Phase zwei weitere Themenbereiche unterschieden: zum einem die Entwicklung von (neuartigen) Systementwicklungsmethoden (Tab. 1 - Themenfeld 5) sowie zum anderen die Unterstützung von (bestehenden) Systementwicklungsmethoden (Tab. 1 - Themenfeld 6). Anschließend wurde analysiert, inwieweit die identifizierten Beiträge geeignet sind, die definierten Forschungsfragen zu beantworten (Abschn. 1). Die Ergebnisse der Klassifizierung und Analyse werden im Folgenden weiter erläutert.

\section{Kultur in der gestaltungsorientierten Forschung}

Im Folgenden werden die Ergebnisse der Literaturanalyse entlang des Ordnungsrahmens aus Abb. 2 vorgestellt und könnten als Grundlage einer kultursensitiven Entwicklungstheorie dienen. Die Zuordnung der Beiträge ist zusammenfassend in Tab. 1 dargestellt und orientiert sich an den einzelnen Phasen bzw. Themenfeldern der Entwicklung erfolgreicher soziotechnischer Systeme, die die
Zeilen der Tabelle ergeben. Sofern gegeben wird jeweils ein Beispiel für jedes Themenfeld näher erläutert.

Damit wird die erste der in Abschn. 1 aufgeworfenen Forschungsfragen adressiert, welche Forschungsergebnisse in den verschiedenen Bereichen erzielt wurden. Darauf aufbauend werden noch offene Forschungslücken zu jedem Themenfeld herausgearbeitet. Damit fokussieren wir die zweite Forschungsfrage, welche Forschungspotenziale bestehen und wie diese erschlossen werden können. Eine vollständige Auflistung der analysierten Beiträge sowie deren Ergebnisse sind in Online-Anhang A beigefügt.

\subsection{Vorphase}

Der Vorphase wurden jene Beiträge zugeordnet, die Forschungsfragen thematisieren, die der tatsächlichen Entwicklung vorgelagert sind. Konkret handelt es sich hierbei um die Identifikation kultursensitiver Informationssysteme für zukünftige Forschungsvorhaben und grundsätzliche Werteunterschiede von Entwicklern.

Themenfeld 1 - Potenziale zukünftiger kultursensitiver Informationssysteme:

Dieses Themenfeld zielt auf die Identifikation von stark kulturell geprägten 


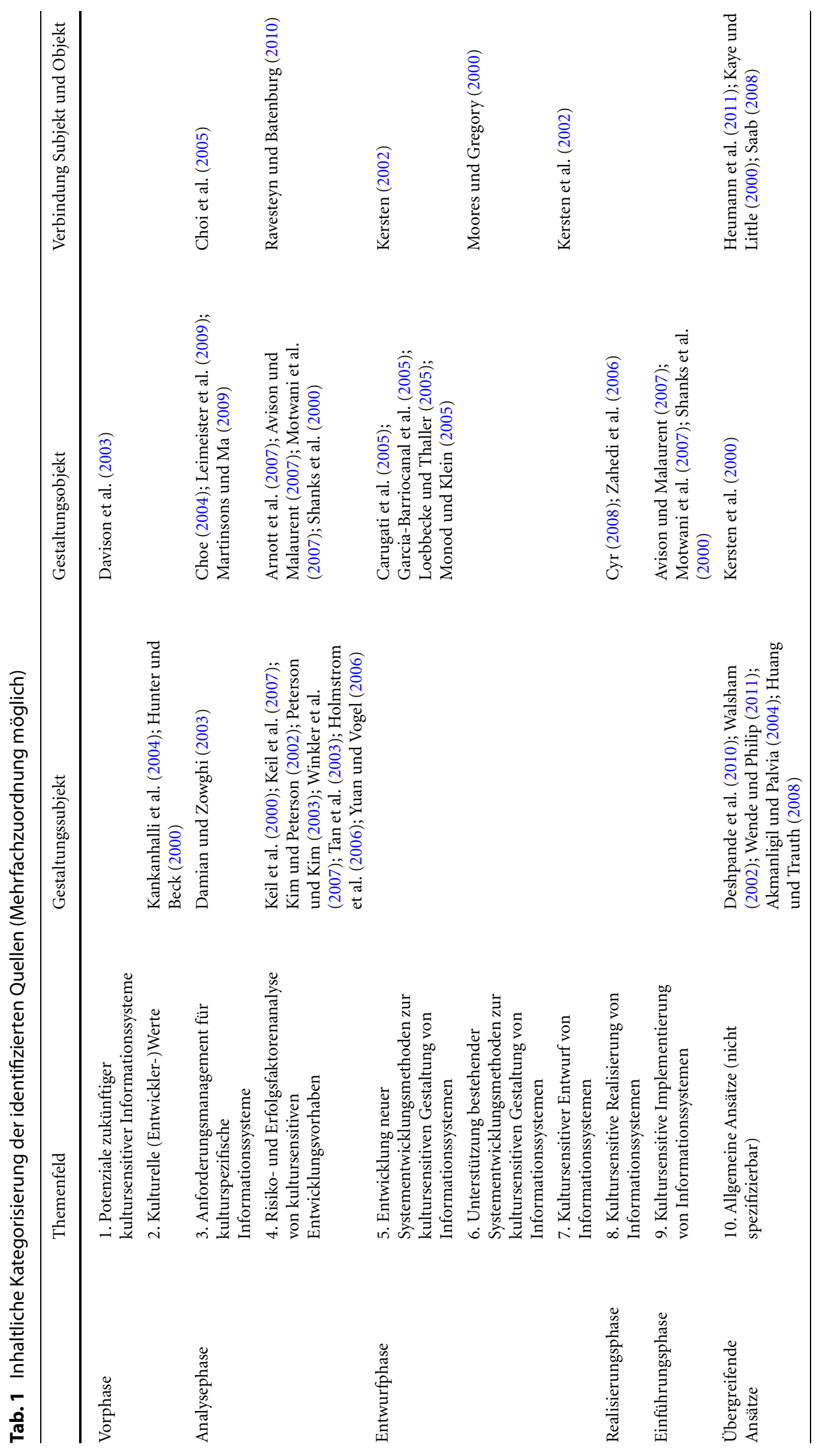


Informationssystemen ab, um zukünftige Forschungsvorhaben zu initiieren. Die kulturellen Aspekte werden daher bereits vor der Systementwicklung konzeptionell oder empirisch untersucht. Davison et al. (2003) ist der einzige Beitrag, der dieser Kategorie zugeordnet werden konnte. In dem konzeptionellen Beitrag werden E-Commerce-Möglichkeiten entlang der früheren Seidenstraße in Zentralasien aufgezeigt. Bezüglich der Infrastruktur besteht in der Seidenstraßenregion ein vergleichsweise gut ausgebautes Mobilfunknetz, weshalb mobilen Endgeräten eine Schlüsselrolle zugesprochen wird. Dennoch ergeben sich aufgrund fehlender Zahlungssysteme Barrieren, da u. a. in Westchina kaum Kreditkarten akzeptiert werden. Hinzu kommen kulturelle Problemstellungen, die sich aus der Vielzahl beteiligter Nationen ergeben (Türkei, Kasachstan, Turkmenistan, Indien, Nepal und China). Die involvierten Regionen sind zudem vergleichsweise arm, woraus sich wirtschaftliche Probleme ergeben. Die Autoren gehen daher davon aus, dass der Aufbau einer funktionsfähigen E-Seidenstraße noch mehrere Jahre dauern wird. $\mathrm{Zu}$ beachten ist, dass dieser Beitrag vergleichsweise allgemein gehalten ist und über keinerlei theoretische Fundierung verfügt.

Da diesem Themenbereich nur ein Beitrag zugeordnet werden konnte, stellen sich sehr allgemeine Forschungsfragen:

- Welche weiteren Bereiche weisen ein hohes Potenzial für kultursensitive Informationssysteme auf?

- Wie kann dieses Potenzial erschlossen werden?

Themenfeld 2 - Kulturelle (Entwickler-) Werte:

Auch dieses Themenfeld ist der tatsächlichen Entwicklung vorgelagert und umfasst die generelle (nationale) kulturelle Prägung von Entwicklern und deren Berücksichtigung bei der Teambildung für Entwicklungsprojekte. Die beiden identifizierten Beiträge nutzen jeweils Hofstedes Kulturdimensionen als theoretischen Ausgangspunkt. Kankanhalli et al. (2004) untersuchen den Zusammenhang zwischen Individualismus/Kollektivismus sowie Maskulinität/Femininität auf die Entwicklerwerte nach Kumar und BjornAndersen (1990). Dabei gelingt es den Autoren den Einfluss der beiden Kulturdimensionen auf die technischen, ökonomischen und sozio-politischen Werte der Entwickler anhand von empirischen Daten aus den USA und Singapur zu belegen. Individualismus und
Maskulinität sind einerseits positiv mit ökonomischen und technischen Werten verbunden, andererseits besteht $\mathrm{zu}$ den sozio-politischen Werten ein negativer Zusammenhang. Diese Ergebnisse sollten bei der Zusammensetzung und dem Management interkultureller Teams berücksichtigt werden. Auch Hunter und Beck (2000) untersuchen kulturelle Entwicklerwerte auf der Ebene des Gestaltungssubjekts. Unter Rückgriff auf die Repertory-Grid-Technik (Kelly 1955 und 1963) wird gezeigt, dass in unterschiedlichen Kulturen unterschiedliche Maßstäbe und Gütekriterien für die Leistungsbeurteilung verwendet werden. Bei dieser qualitativen Arbeit wurden verschiedene Konstrukte mithilfe von Interviews beurteilt. Als Beispiel werden anhand von Hofstedes Kulturdimensionen Kanada und Singapur ausgewählt. Dabei wird unter anderem herausgearbeitet, dass Systemanalysten in Singapur einen technokratischeren Ansatz verfolgen, wobei Expertise genutzt wird, um eine dominante Rolle in einem IS-Projekt einzunehmen. Im Unterschied dazu nehmen die kanadischen Systemanalysten eher eine beratende Rolle ein und versuchen den Kunden stärker bei der Arbeit mit einzubeziehen. Diesen Rollen entsprechend ergibt sich eine unterschiedliche Erwartungshaltung an die zu erbringende Leistung. Der Schwerpunkt des Beitrags liegt allerdings auf der Entwicklung einer Methodik und weniger auf der dezidierten Ergebnisanalyse.

Vor diesem Hintergrund erscheinen die folgenden Forschungsfragen in diesem Themenfeld bedeutsam:

- Welche Auswirkungen haben unterschiedliche kulturelle (Entwickler-) Werte auf das Gestaltungsobjekt?

- Lassen sich die Ergebnisse auf andere Länder mit ähnlichen Ausprägungen bezüglich der Kulturdimensionen verallgemeinern (z. B. China bezüglich des Kollektivismus)?

\subsection{Analysephase}

Nach Stahlknecht und Hasenkamp (2004) gliedert sich die Analysephase in eine Ist-Analyse, bei der das bestehende System untersucht wird, und eine Sollanalyse, bei der ein erstes Sollkonzept entwickelt wird. Dieser Kategorie konnten zwei Themenfelder zugeordnet werden: Zum einen das Anforderungsmanagement, zum anderen die Erfassung und Berücksichtigung von Risiko- und Erfolgsfaktorenanalyse.
Themenfeld 3 - Anforderungsmanagement für kulturspezifische Informationssysteme: In diesem Themenbereich sind jene Beiträge zusammengefasst, die kulturspezifische Hinweise zur Erhebung oder Abbildung bzw. zu kulturkreisspezifischen Anforderungen thematisieren. Die identifizierten Beiträge decken sowohl Gestaltungssubjekt, -objekt und deren Beziehung zueinander ab. Bezüglich des Gestalters kommen Damian und Zowghi (2003) zu dem Ergebnis, dass das Anforderungsmanagement bei globaler Softwareentwicklung in Australien und den USA durch Konflikte aufgrund kultureller Unterschiede beeinträchtigt wird.

Choe (2004) zeigt kulturbedingte Unterschiede hinsichtlich der Funktionalitätsanforderungen von Informationssystemen im Rechnungswesen auf, die bei deren Design berücksichtigt werden sollten. Koreanische Unternehmen legen mehr Wert auf eine flexible Performance, während für australische Unternehmen insbesondere Leistungsqualität und traditionelle Informationen zur Kostenkontrolle von Bedeutung sind.

Leimeister et al. (2009) wiederum verdeutlichen, dass die Anforderungen von CIOs an RFID mit unterschiedlichen strategischen Zielen verbunden sind. Während bei deutschen CIOs Qualitätsverbesserungen, die Reduzierung oder Automatisierung von menschlicher Arbeitskraft, eine Verringerung gefälschter Produkte und eine Verbesserung des Kundenservice im Mittelpunkt stehen, ist die Zielsetzung bei italienischen CIOs primär auf die Verringerung von inkonsistenten Warenbeständen, die Optimierung der Lagerhaltung und die Verbesserung des Kundenservice ausgerichtet. Als Treiber dieser Zielsetzungen konnten die Faktoren Erfahrung mit RFID, Unternehmensgröße und wahrgenommenes Potenzial der Technologie ermittelt werden. Hofstedes Kulturdimensionen wurden genutzt, um Unterschiede zwischen Deutschen und Italienern bezüglich dieser Einflussgrößen zu erklären. So wird beispielsweise die geringere Erfahrung mit RFID in Italien auf die höhere Unsicherheitsvermeidung nach Hofstede zurückgeführt.

Choi et al. (2005) identifizieren 52 Attribute, die einen Einfluss auf die Nutzung von mobilen Datenservices ausüben. Darüber hinaus werden elf kritische Attribute herausgearbeitet, die in einem direkten Zusammenhang zur Nutzerkultur aufweisen. Als theoretische 
Grundlage werden auch hier die Kulturdimensionen von Hofstede herangezogen (Unsicherheitsvermeidung und Individualismus/Kollektivismus). Darüber hinaus werden von Hall und Hall (1990) die Kulturdimensionen des Kontexts und die Zeitwahrnehmung genutzt. Als Beispiel wird der Kontext in Korea und Japan durch Präferenzen für eine größere Anzahl an Inhalten sowie Symbolen im Übersichtsdesign, die Varianz an Schriftfarben und Schriftgrößen berücksichtigt, während in Finnland weniger Inhalte sowie weniger Symbole im Übersichtsdesign und die Varianz an Schriftfarben favorisiert werden. Diese Anforderungsunterschiede sollten beim Design von mobilen Datenservices in unterschiedlichen Kulturkreisen genutzt werden, um eine höhere Akzeptanz zu erreichen. Bezüglich der inhaltlichen Einordnung zielen Choi et al. (2005) vornehmlich auf das Gestaltungssubjekt ab. Da jedoch potenzielle Nutzer befragt wurden, stellt der Beitrag dennoch eine Verbindung beider Ebenen dar.

Martinsons und Ma (2009) analysieren, inwieweit sich unterschiedliche chinesische Subkulturen bezüglich der Informationsethik von Managern unterscheiden. Dabei werden statt den von Hofstede üblichen Kulturdimensionen die Dimensionen der Generationssubkulturtheorie (generational subculture theory) nach Strauss und Howe (1991) sowie die Lebenszyklustheorie nach Erikson (1997) herangezogen. Diese Ansätze gehen nicht von einer homogenen Kultur aus, sondern erklären altersbedingte Unterschiede in der Kultur einer Nation. Eigeninteresse, soziale Beziehungen, Regel und Gesetze, Mehrheitsrecht sowie Gleichheit und Fairness werden als Indikatoren für Moralunterschiede herangezogen. Die Ergebnisse lassen auf eine westlich geprägte, eine traditionelle und eine moderne chinesische Kultur schließen, die entsprechende Anpassungen bezüglich des Anforderungsmanagements erfordern. Diese Arbeit wirft zudem Zweifel am Kulturverständnis von Hofstede und der GLOBE-Studie auf (Abschn. 2), die von nur "einer“ chinesischen Kultur ausgehen.

Zusammenfassend lässt sich feststellen, dass kulturbedingt unterschiedliche Anforderungen an Informationssysteme bestehen, die bei der Entwicklung berücksichtigt werden sollten. Darüber hinaus wird auch das Anforderungsmanagement selbst durch Kultur beeinflusst und wird folglich in verschiedenen Kulturkreisen unterschiedlich ausgeführt. In diesem Zusammenhang stellt sich die Frage, ob sich die vorliegenden Ergebnisse tatsächlich auf den gesamten nationalen Kulturkreis verallgemeinern lassen oder ob die von Martinsons und Ma (2009) vertretene Annahme zutreffend ist, dass innerhalb einer Nation Subkulturen bestehen, deren Anforderungen sich erheblich unterscheiden können. Betrachtet man die Größe und Vielschichtigkeit einiger Länder wie China oder Indien und die Unterschiede zwischen den Generationen sowie Land- und Stadtbevölkerung, so erscheint eine Einordnung nach Hofstede für eine gesamte Nation zu ungenau.

Basierend auf den Ausführungen erscheinen die folgenden Forschungsfragen relevant:

- Inwieweit bestehen in Nationen nationale Subkulturen und wie stark unterscheiden sich diese gegenüber Hofstedes Dimensionen?

- Lassen sich die identifizierten Anforderungsunterschiede tatsächlich auf die zur Erklärung genutzten Kulturdimensionen zurückführen?

Themenfeld 4 - Risiko- und Erfolgsfaktorenanalyse von kultursensitiven Entwicklungsvorhaben:

Diesem Themenfeld wurden Beiträge zugeordnet, die sich mit der Analyse kritischer Faktoren von Entwicklungsvorhaben auseinandersetzen. Die Analyse von Risikofaktoren erfolgt zumeist begleitend oder zyklisch im Entwicklungsprozess (Boehm 1986). Sofern möglich wurden die Beiträge daher zusätzlich einer konkreten Phase des Entwicklungsprozesses zugeordnet. Kulturelle Faktoren, die für den Erfolg von Informationsund Kommunikationstechnologie (IKT)Entwicklungsvorhaben relevant sind, konnten auf sämtlichen Kulturebenen herausgearbeitet werden. Aufgrund der zahlreichen Beiträge stellt auch dieser Themenbereich einen Schwerpunkt dar, der einer detaillierten Erläuterung bedarf.

Hinsichtlich des Bezugsobjekts dominiert in diesem Themenfeld das Gestaltungssubjekt, konkret die Gestaltungsmitglieder (Abb. 2), die Ausrichtung der Beiträge. Im Mittelpunkt der Forschung steht zumeist die kooperative Entwicklung von Informationssystemen in unterschiedlichen nationalen Kulturräumen. Ein oftmals identifiziertes Phänomen im asiatischen Raum ist dabei das Vermeiden des Gesichtsverlusts bei der Kommunikation von Problemen (Winkler et al. 2007; Keil et al. 2007; Tan et al. 2003; Yuan und Vogel 2006). Dies kann gerade bei Offshore-Projekten, bei denen Gestalter aus verschiedenen Ländern zusammenarbeiten, zu Problemen führen. Peterson und Kim (2003) untersuchen die kulturellen Unterschiede, die in der Wahrnehmung von Erfolgsfaktoren bei Softwareentwicklern aus den USA, Korea und Japan bestehen. So werden unzureichende Nutzerbeteiligung und unzureichende Erfahrung in Korea als problematischer wahrgenommen. Zudem stellen auch Projektzieldefinitionen und verpasste Deadlines in Korea ein größeres Problem dar, welches zu Entwicklungsfehlschlägen führt. In Gegensatz dazu wiesen die Entwickler in Japan und USA ähnliche Ergebnisse auf und beurteilten die Erfolgsfaktoren insgesamt positiver. Ebenso arbeiten Keil et al. (2000) heraus, dass das Scheitern von Softwareentwicklungsprojekten in verschiedenen Kulturkreisen unterschiedlich beurteilt wird. Konkret werden Finnland, die Niederlande und Singapur verglichen, wobei insbesondere auf den Umgang mit Sunk Costs und die Risikotendenz von Entscheidern abgezielt wird. Während die Höhe der Sunk Costs in allen Kulturkreisen die Akteure dazu animiert, ein höheres Risiko einzugehen, konnte gezeigt werden, dass die Risikotendenz in Ländern mit einem niedrigen Grad an Unsicherheitsvermeidung wie bspw. Singapur dazu führen, dass die Risikowahrnehmung verringert wird und kritische Projekte länger fortgesetzt werden.

Darüber hinaus wurden diesem Themenfeld auch die Beiträge von Avison und Malaurent (2007), Motwani et al. (2007) und Shanks et al. (2000) zugeordnet, die jeweils kritische Erfolgsfaktoren für die Einführung von Enterprise-Resource-Planning(ERP-)Systemen analysieren. Aufgrund des inhaltlichen Schwerpunkts werden diese in Abschn. 4.5 (Einführungsphase) detailliert betrachtet.

Im Beitrag von Ravesteyn und Batenburg (2010) wird der Zusammenhang zwischen dem Gestalter auf der Seite des Gestaltungssubjekts und dem Menschen auf Seite des Gestaltungsobjekts untersucht. Dabei werden kulturelle Unterschiede bei der Implementierung von Business-Process-Management-Systemen untersucht, die von diesen beiden Ebenen beeinflusst werden. Statt der 
Verwendung allgemeiner Kulturdimensionen wurden Probanden aus Nordeuropa und den Anglo-amerikanischen Raum Fragen gestellt, die u. a. Rückschlüsse auf das Verständnis des Geschäftsprozessmanagementkonzepts, die Managementunterstützung und die Expertise beim Change Management umfassen. Es wurde deutlich, dass sich die kritischen Erfolgsfaktoren für die Implementierung von Business-ProcessManagement-Systemen kulturunabhängig fünf Bereichen zuordnen lassen ( $\mathrm{Ma}$ nagement der Organisation und der Prozesse, Messung und Kontrolle, Implementierung und Change Management, Architektur, Lösungsentwicklung). Allerdings unterscheidet sich die Relevanz einzelner Faktoren in diesen Bereichen deutlich. So werden die Verständlichkeit des generellen Konzepts und der Prozesse, deren Beziehung zueinander sowie eine hohe Unterstützung durch das Management, insbesondere in Nordeuropa als wichtig erachtet.

Weiterhin konnten Beiträge identifiziert werden, die das Gestaltungssubjekt adressieren und dabei Aspekte der nationalen und der organisationalen Kultur verbinden. Tan et al. (2003) kommen zu dem Ergebnis, dass die Entscheidung, ob schlechte Nachrichten zu einem Softwareprojekt kommuniziert werden, in Ländern mit einem hohen $\mathrm{Maß}$ an Kollektivismus primär durch das organisationale Klima beeinflusst wird. Demgegenüber kommen Holmstrom et al. (2006) zu dem Ergebnis, dass Unterschiede in der sozio-kulturellen Distanz globale Entwicklungsprojekte beeinträchtigen. Bei der sozio-kulturellen Distanz handelt es sich um ein von Ågerfalk et al. (2005) übernommenes Konstrukt, das Aspekte der nationalen und organisationalen Kultur vereint und angibt, inwieweit ein Akteur die Werte und normativen Handlungen anderer Akteure versteht. Darüber hinaus werden Einflüsse der temporalen und geografischen Distanz (ebenso von Ågerfalk et al. 2005) untersucht. Im Ergebnis der Fallstudienbetrachtung werden insbesondere Kommunikationsschwierigkeiten angeführt, die bspw. die Verwendung unterschiedlicher Sprachen und Fachbegriffe beinhalten sowie Unterschiede in der Einschätzung des benötigten Zeitbedarfs. Doch obgleich die gleichen Probleme in vier untersuchten Unternehmen auftraten, werden unterschiedliche Lösungsstrategien verfolgt. Auf theoretischer Ebene ergibt sich durch die Verbindung verschiedener Kulturarten (national und organisational) in den Distanzen ein zusätzlicher Erklärungsgehalt gegenüber klassischen Erklärungsansätzen wie bspw. Hofstede.

Die zahlreichen Beiträge zum Thema Erfolgsfaktoren weisen deutliche Gemeinsamkeiten auf. Der Gesichtsverlust in asiatischen Ländern, Kommunikations- und Koordinationsprobleme, Machtdistanz und Unsicherheitsvermeidung sowie Kollektivismus stehen dabei im Mittelpunkt. Die Kulturdimensionen werden dabei oftmals als Begründung beobachteter Phänomene herangezogen. Widersprüche zwischen den Ergebnissen konnten nicht identifiziert werden. Allerdings betonen die Beiträge die Relevanz einzelner Faktoren mitunter unterschiedlich.

Die Analyse der Beiträge in diesem Themenfeld führte $\mathrm{zu}$ folgenden Forschungsfragen:

- Inwieweit lässt sich das Konzept der sozio-kulturellen Distanz auf andere Themenbereiche übertragen?

- Warum treten Ausprägungen bei den beobachteten Phänomenen wie bspw. „Verständlichkeit des generellen Konzepts und der Prozesse“ oder „unzureichende Erfahrung“ in einzelnen Kulturkreisen auf?

\subsection{Entwurfsphase}

In der Entwurfsphase wird das Informationssystem anhand der zuvor bestimmten Soll-Anforderungen entwickelt (Stahlknecht und Hasenkamp 2004). Dabei wird unter Verwendung von Systementwicklungsmethoden ein Systementwurf erstellt. Dieser Phase konnten Beiträge zugeordnet werden, die die Entwicklung und Unterstützung von kultursensitiven Systementwicklungsmethoden thematisieren sowie Beiträge, die den kultursensitiver Systementwurf direkt adressieren.

Themenfeld 5 - Entwicklung neuer Systementwicklungsmethoden zur kultursensitiven Gestaltung von Informationssystemen:

In Beiträgen, die diesem Themenbereich zugeordnet wurden, werden neue Systementwicklungsmethoden und Architekturen vorgestellt, die Kultur explizit berücksichtigen. Zahlreiche Beiträge wurden identifiziert, die als Aufgabe des Gestaltungsobjekts die Speicherung von Kultur im Sinne von kulturellem Erbe
(Culture Heritage) durch Informationssysteme thematisieren (Carugati et al. 2005; Garcia-Barriocanal et al. 2005; Loebbecke und Thaller 2005; Monod und Klein 2005). Diese Beiträge sind überwiegend gestaltungsorientiert und beinhalten Methoden und Architekturen zur Entwicklung entsprechender Systeme. So stellen Loebbecke und Thaller (2005) eine Referenzarchitektur vor, die die Digitalisierung von Kulturobjekten (z. B. dem Inventar eines Museums) unterstützt und online verfügbar macht und verdeutlichen deren Anwendung anhand von zwei Fallbeispielen. Da diese Beiträge eine kulturelle Aufgabenstellung mit einer konkreten Technik adressieren, lassen sie sich den Elementen Aufgabe und Technik des Ordnungsrahmens zuordnen. Diese Ausrichtung auf diese beiden Elemente zeigt allerdings auch deutlich, dass das soziale Subsystem bisher nicht thematisiert wurde und sich daher für zukünftige Forschung anbietet. Es konnten keine Studien identifiziert werden, die untersuchen, wie bspw. kulturelle Präferenzen die Digitalisierung von kulturellem Erbe beeinflussen.

Ein weiterer Beitrag beschreibt eine neuartige Methodik, um Informationssysteme an die Kultur der Nutzer anzupassen und ist folglich der Verbindung von Gestaltungsobjekt und Gestaltungssubjekt zuzuordnen (Kersten 2002). Er verdeutlicht anhand der Entwicklung von E-Business Systemen, dass es bei der Berücksichtigung kultureller Artefakte nicht ausreichend ist, diese durch ein entsprechend angepasstes Design der Oberfläche (z. B. Sprachanpassungen) $\mathrm{zu}$ realisieren. Stattdessen müsste auch die Kernapplikation entsprechend den Kulturpräferenzen angepasst werden. Die Wichtigkeit, die Aspekten wie Offenheit des Systems, Flexibilität, Stabilität und Benutzerfreundlichkeit in einer Kultur zugesprochen werden, sind Beispiele für Eigenschaften, die mit kulturellen Werten verbunden sind und sich unter anderem in der Wahl eines Windows- oder Linux-basierten Betriebssystems artikulieren können. Dabei werden verschiedene Kulturformen (national, organisational) vermengt, um anhand von Beispielen zu erläutern, wie die Entwicklung der verschiedenen Ebenen des E-Business Systems einschließlich des Softwarekerns an die Kultur angepasst werden können. Zudem werden neuartige Entwicklungsparadigmen wie die aspekt- oder subjektorientierte Programmierung vorgeschlagen, um kulturelle Eigenschaften bei der Applikationsentwicklung zu realisieren. 
Es ergeben sich die folgenden Forschungsfragen:

- Welche nationalen Kulturdifferenzen weisen Systeme auf, die kulturelles Erbe speichern?

- Lassen sich die von Kersten (2002) konzeptionell hergeleiteten Erfordernisse hinsichtlich der Anpassung der Kernapplikation empirisch bestätigen?

Themenfeld 6-Unterstützung bestehender Systementwicklungsmethoden zur kultursensitiven Gestaltung von Informationssystemen:

Diese Kategorie umfasst jene Beiträge, die bereits existierende Methoden thematisieren, um kulturelle Aspekte bei der Entwicklung von Informationssystemen $\mathrm{zu}$ unterstützen, also Kulturaspekte des soziotechnischen Systems methodisch adäquat zu adressieren.

Moores und Gregory (2000) untersuchen kulturbedingte Probleme bei der Nutzung einer Soft Systems Methodology (SSM). Beim SSM handelt es sich um eine Analyse- und Designmethodik, die es ermöglicht, Problemsituationen zu strukturieren und dabei die menschlichen Akteure sowie deren Kultur zu berücksichtigen (Checkland 1981). Am Beispiel von China (hier: Hong Kong) werden drei kulturelle Problembereiche in der Softwareentwicklung aufgezeigt: So werden Gruppendiskussionen vermieden, Interviews werden in mehreren Sprachen durchgeführt, woraus Kommunikationsprobleme resultieren und aufgrund einer hohen Personalfluktuation im Management ergeben sich Schwierigkeiten bei der konsequenten Berücksichtigung von Stakeholder-Interessen.

Die folgenden Forschungsfragen konnten identifiziert werden:

- Wie können Entwicklungsmethoden verschiedene Subkulturen bei der Entwicklung des Systems berücksichtigen?

- Welche Entwicklungsmethoden sind für welche Kulturkreise geeignet?

- An welchen Kulturfaktoren sollte die Auswahl einer bestimmten Methode erfolgen?

Themenfeld 7 - Kultursensitiver Entwurf von Informationssystemen:

In dem Beitrag von Kersten et al. (2002) wird der Einfluss von Kultur auf die Oberflächen- und Kernapplikationsgestaltung untersucht. Der Beitrag weist dabei eine inhaltliche Nähe zu Kersten (2002) auf (siehe Themenfeld 5). Es wird problematisiert, dass bei der Softwaregestaltung oftmals eine Reduzierung von Kultur auf Sprache und Symbole erfolgt bzw. die instrumentelle Theorie von Technologie vorliegt. Diesem Ansatz nach wird eine internationale Software erstellt, deren Oberfläche national angepasst wird. Im Gegensatz da$\mathrm{zu}$ steht ein ganzheitliches Kulturverständnis bzw. die sogenannte substanzielle oder kritische Theorie von Technologie. Da Softwareapplikationen auf die Nutzerinteraktion zugeschnitten werden, ist es nach diesem weitreichenden Kulturverständnis nicht ausreichend nur die Benutzeroberfläche anhand der bestehenden Kulturen anzupassen. Stattdessen sollte auch eine Anpassung des Softwarekerns angestrebt werden. Als Beispiel wird ein Entscheidungsunterstützungssystem in Frankreich angeführt. Da hier nach Hofstedes Dimensionen eine hohe Machtdistanz und eine feminine Orientierung vorliegen, ergibt sich eine hohe Wertschätzung von Lebensqualität, Beziehungen und Autorität. Aus diesem Grund sollte das System die Autorität des Entscheidungsträgers berücksichtigen und erhebliche Freiheitsgrade bieten. In dem Systementwurf sollte dies entsprechend berücksichtigt werden. Kersten et al. (2002) schlagen dafür einen Ansatz vor, bei dem zwischen kulturabhängigen und kulturunabhängigen Komponenten unterschieden wird, um dann, einem modularen Gestaltungsansatz entsprechend, gezielte Anpassungen vorzunehmen. Die theoretische Verknüpfung von Kultur- und Technikverständnis sticht aus den identifizierten Beiträgen heraus und stellt einen geeigneten Ansatzpunkt für zukünftige Forschungsvorhaben dar. Da neben dem Nutzer auch spezielle Gestaltungstechniken berücksichtigt werden, wurde der Beitrag beiden Elementen zugeordnet.

Folgende Forschungsfragen erscheinen besonders bedeutsam:

- Inwieweit lässt sich das von Kersten et al. (2002) entwickelte theoretische Konzept praktisch umsetzen und sind die daraus resultierenden Systeme tatsächlich zielführender?

- Inwieweit beeinflusst der kulturelle Hintergrund des Gestalters den Systementwurf?

\subsection{Realisierungsphase}

Dieser Phase wurden Beiträge zugeordnet, die sich mit der Umsetzung zuvor spezifizierter Systeme beschäftigen.
Themenfeld 8 - Kultursensitive Realisierung von Informationssystemen:

Zur Realisierung konnten nur Beiträge identifiziert werden, die sich mit der Gestaltung von Webseiten beschäftigen. Diese bauen auf der Usability-Literatur auf und berücksichtigen Hofstedes Kulturdimensionen. Am Beispiel des Onlineshopping untersuchen Cyr (2008), inwieweit sich Informationsdesign, Navigationsdesign und visuelles Design auf Vertrauen und Zufriedenheit auswirken. Diese beiden Faktoren beeinflussen die sogenannte E-Loyalität definiert als die Absicht, eine Webseite erneut zu besuchen oder diese bei zukünftigen Kaufentscheidungen in Erwägung zu ziehen. E-Loyalität stellt daher ein wesentliches Merkmal von Kundenbindung dar. Die Ergebnisse verdeutlichen, dass Vertrauen insbesondere in Ländern mit einer hohen Unsicherheitsvermeidung einen wichtigen Einflussfaktor der E-Loyalität ist. Aus diesem Grund ist dieser Faktor in Deutschland und China relevanter als in Kanada. Weiterhin wird aufbauend auf der bestehenden Literatur ein hohes Maß an Kollektivismus in China mit einer Vorliebe für kontrastreiche Farben und visuelle Effekte begründet, während in Deutschland aufgrund der individualistischen Prägung ein logisches und strukturiertes Layout präferiert wird (Sun 2001). Die Ergebnisse stützen diese Hypothese, da nur in China ein signifikanter Zusammenhang zwischen visuellem Design und Vertrauen festgestellt werden kann. Insgesamt scheinen die verschiedenen Designs allerdings kulturübergreifend geeignet, um Vertrauen und Zufriedenheit und darüber indirekt die E-Loyalität zu erklären.

Im Gegensatz dazu untersuchen Zahedi et al. (2006) den Einfluss zwischen Kultur und Hofstedes Kulturdimension Maskulinität-Femininität zur Steigerung der Effizienz von Webdokumenten. Kultureller Inhalt sollte dabei bewusst an die Werte der Zielgruppe angepasst werden, da sonst kulturelle Botschaften enthalten sein könnten, die die Kommunikation verzerren. Maskulin geprägte Webdokumente zeichnen sich demnach durch ein dichtes Maß an Fakten aus und benutzen Worte, die typischerweise mit männlichen Eigenschaften verbunden werden. Demgegenüber zeichnen sich feminine Webdokumente durch Aspekte verbunden mit Wohltätigkeit, Gemeinschaft, Teilen und zwischenmenschliche Beziehungen aus. Zahedi 
et al. (2006) arbeiten diese stereotypischen Attribute aus verschiedenen Webseiten heraus und zeigen auf, dass zahlreiche Webseiten ein für die Zielgruppe ungeeignetes Design nutzen. Obgleich es sich um eine typische nationale Kulturdimension handelt, wird jedoch kein Zusammenhang zu diesem Forschungsbereich hergestellt. Es bleibt daher offen, ob sich die Ergebnisse grundsätzlich auf maskulin/feminin geprägte $\mathrm{Na}$ tionen übertragen lassen. Bezüglich der inhaltlichen Einordnung adressieren beiden Ansätze das Gestaltungsobjekt und insbesondere den Menschen.

Aus den Beiträgen lassen sich die folgenden Forschungsfragen ableiten:

- Inwieweit beeinflusst die kulturelle Prägung des Gestalters (z. B. bezüglich Designpräferenzen) die Realisierungsphase?

- Lassen sich die Ergebnisse von Zahedi et al. (2006) auf maskulin bzw. feminin geprägte Nationen übertragen?

- Welche Folgen ergeben sich bei Nichtbeachtung von kulturellen Unterschieden bei der Realisierung von Kernapplikationen?

\subsection{Einführungsphase}

Diese Phase umfasst die konkrete Einführung eines zuvor spezifizierten Systems. Konsequenterweise wurden dieser Phase Beiträge zugeordnet, die sich mit den kulturellen Herausforderungen bei der Implementierung auseinandersetzen.

Themenfeld 9 - Kultursensitive Implementierung von Informationssystemen:

Auf der Ebene des Gestaltungsobjekts identifizieren Avison und Malaurent (2007) in China kulturbedingte Probleme, die im beschriebenen Fall zum Scheitern eines Enterprise-Resource-Planning-(ERP-) Einführungsprojekts eines französischen Unternehmens führten. Die aufgezeigten Probleme umfassten die Sprache und das Kommunikationsverhalten der Nutzer (bspw. durch Gesichtsverlust oder Verständnisprobleme bei Anweisungen in englischer Sprache), wirtschaftliche Aspekte (unerwartete lokale Berichtspflichten) sowie strukturelle Faktoren (Respekt vor der Unternehmenshierarchie). Die Autoren schlussfolgern daher, dass der Einsatz von globalen Vorlagen zur Unterstützung von Systemeinführungen negative Folgen haben kann.

Auch Motwani et al. (2007) thematisieren den Zusammenhang zwischen dem
Menschen und einer konkreten Technik auf der Ebene des Gestaltungsobjekts. Kulturelle Unterschiede bei der Einführung von ERP-Systemen zwischen Indien und den USA ergeben sich danach insbesondere durch Hofstedes Dimensionen der Machtdistanz und Individualismus/Kollektivismus. Die Machtdistanz führt dazu, dass das Top-Management in Indien wesentlich stärker eingebunden ist, einseitig Meilensteine setzt und deren Einhaltung überwacht. Im Unterschied dazu wird in den USA aufgrund der geringeren Machtdistanz eher ein teamorientierter Ansatz gewählt, bei dem wesentliche Entscheidungen kollaborativ getroffen werden. Darüber hinaus werden externe Experten in kollektivistischen Gesellschaften wie Indien leichter akzeptiert, wohingegen individualistisch geprägte Kulturen wie die USA eher auf die technische Expertise der eigenen Mitarbeiter vertrauen. Darüber hinaus bestehen jedoch auch Erfolgsfaktoren wie ein professionelles Projektmanagement, die kulturübergreifend wirken. Auch Shanks et al. (2000) kommen zu dem Ergebnis, dass bei der Einführung von ERP-Systemen das soziale Subsystem gerade durch die Kulturdimension der Machtdistanz und Individualismus/Kollektivismus beeinflusst wird. Dies wird anhand Fallstudien in China und Australien darauf zurückgeführt, dass in Ländern mit einem hohen Grad an Machtdistanz und Kollektivismus wie China weniger Ressourcen eingesetzt werden müssen, um die Menschen von einer Veränderung ihres Verhaltens zu überzeugen.

$\mathrm{Da}$ alle Beiträge in diesem Themenfeld Risikofaktoren untersuchen, wurden die Beiträge auch Themenbereich 4 zugeordnet.

Bezüglich dieses Themengebietes erscheinen die folgenden Fragestellungen besonders relevant.

- Inwieweit beeinflusst die kulturelle Prägung des Gestalters (z. B. bezüglich Designpräferenzen) die Einführungsphase?

- Lassen sich bei globalen Vorlagen zur Systemeinführungen kulturunabhängige und kulturabhängige Komponenten unterscheiden?

- Lassen sich die wiederholenden Beobachtungen hinsichtlich der Kulturdimensionen Machtdistanz sowie Individualismus/Kollektivismus verallgemeinern?

\section{6 Übergreifende Ansätze}

Themenfeld 10 - Allgemeine Ansätze (nicht spezifizierbar):

Zahlreiche weitere Beiträge konnten keinem der zuvor thematisierten Bereiche zugeordnet werden (Online-Anhang A, Tab. 1). Hierbei handelt es sich um vergleichsweise generelle Ansätze, die sich mit dem gesamten Entwicklungsprozess beschäftigen. Deshpande et al. (2010) zeigen beispielsweise auf nationaler Ebene Techniken zum Umgang mit kulturellen Unterschieden bei der globalen Softwareentwicklung auf und veranschaulichen am Beispiel Indiens, wie diese Unterschiede als Vorteile genutzt werden können. Indien beinhaltet eine Vielzahl von Subkulturen, die allen durch die mehr als 850 gesprochenen Sprachen und mehr als 1.600 Dialekte in Verbindung mit einer Vielzahl von Religionen und lokalen Bräuchen ergeben und damit eine erhebliche Diversität aufweisen. Daher mussten Projektmanager Strategien entwickeln, um mit dieser Vielfalt umzugehen. Ein Beispiel stellt in diesem Zusammenhang die Bereitstellung von BackupTeams dar, die permanent auftretende Ausfälle aufgrund von unterschiedlichen Feiertagen auffangen und dadurch einen 24 Stunden Support an 365 Tagen ermöglichen. Ebenso werden alle Teammitglieder hinsichtlich kultureller Unterschiede sensibilisiert. In speziellen Trainings werden dazu unter anderem Hofstedes Kulturdimensionen thematisiert, Dies führt dazu, dass sowohl die Zusammenarbeit im Team als auch bezüglich der Mandanten verbessert wird.

Demgegenüber zielen Heumann et al. (2011) mit ihrem Beitrag auf die Verbindung zwischen Gestaltungsobjekt und Gestaltungssubjekt ab, indem die Wechselwirkung zwischen der Kultur des Gestalters und der Wahl der Kontrollmechanismen des Kunden in ISOffshoring-Projekten untersucht werden. Dabei werden theoretische Konzepte aus der Kulturforschung mit der Kontrolltheorie kombiniert. Eine Besonderheit besteht hierbei darin, dass die Autoren die gewählten Kulturdimensionen Machtdistanz, Individualismus/Kollektivismus, Unsicherheitsvermeidung, Aktivität/Passivität nach Triandis (1982) sowie das mono-/polychrone Zeitverständnis nach Hall und Hall (1990) mittels eines Fragebogens selbst erheben und damit auf der individuellen Ebene auswertbar machen. Die Ergebnisse veranschaulichen, dass die Machtdistanz die informale Kontrolle beeinflusst, 
wobei sich zwei gegenseitige Effekte ergeben. Während eine hohe Machtdistanz verstärkt auf soziale Kontrollmechanismen wie Rituale, Zeremonien setzt, um Differenzen in den Zielvorstellungen zu reduzieren, wird bei einer geringen Machtdistanz verstärkt Selbstkontrolle eingesetzt. Diese Ergebnisse erscheinen plausibel, da diese Kontrollmechanismen ein höheres Maß an Autonomie benötigen, welches in Kulturen mit einem hohen Maß an Machtdistanz aufgrund der klaren Strukturen und der Erwartungshaltung an Vorgesetzte nicht gegeben ist. Dennoch ist der Einfluss der nationalen Kultur des Lieferanten insgesamt vergleichsweise gering.

Bezüglich dieses Themenbereichs wurden die folgenden Forschungsfragen herausgearbeitet:

- Lassen sich die Ergebnisse von Deshpande et al. (2010) zur gezielten Nutzung kultureller Unterschiede auch auf andere Kulturräume mit weniger Diversifikation übertragen?

- Lassen sich die oftmals als Erklärung für kulturelle Unterschiede herangezogenen Dimensionen von Hofstedes auch empirisch als kausale Einflussfaktoren nachweisen (wie bei Heumann et al. 2011)?

- Lassen sich aus dem Konstrukt der Aktivität/Passivität nach Triandis (1982) weitere kulturelle Unterschiede bei der Systemgestaltung ableiten?

\section{Implikationen}

In diesem Abschnitt werden zunächst die eingangs in Abschn. 1 formulierten Forschungsfragen 1 und 2 beantwortet, wobei die zuvor herausgearbeiteten Ergebnisse zusammengefasst werden (Abschn. 5.1). Anschließend werden im Sinne der dritten Forschungsfrage die Zusammenhänge zwischen den in der Literatur verwendeten Kulturtheorien und der Theorien zur Gestaltung von Informations- und Kommunikationssystemen betrachtet (Abschn. 5.2).

\subsection{Verdichtung der Ergebnisse}

Zusammenfassend lassen sich die Ergebnisse zu zwei zentralen Feldern verdichten: A.) Auf der Seite des Gestaltungssubjekts steht die Frage im Vordergrund, wie interkulturelle Entwicklerteams geführt werden sollen. B.) Demgegenüber dominiert auf Seite des Gestaltungsobjekts die Frage, wie in un- terschiedlichen Kulturkreisen Informationssysteme als soziotechnische Systeme ausgestaltet werden sollen. Unsere Systematisierung (Abb. 2, Tab. 1) bildet für beide Fragestellungen jeweils den aktuellen Forschungsstand ab und veranschaulicht darüber hinaus verschiedene Forschungspotenziale. $\mathrm{Zu} \mathrm{A.)} \mathrm{be-}$ steht eine Vielzahl von Ergebnissen. Diese beschreiben jedoch oftmals nur Einzelphänomene. Hier bieten sich insbesondere eine Synthese der Ergebnisse sowie die Analyse der externen Validität an. Demgegenüber besteht zu B.) noch erheblicher Forschungsbedarf. Die bisherigen Konzepte konzentrieren sich im Wesentlichen nur auf die Erfassung von kulturbedingten Unterschieden. Methoden zur Entwicklung entsprechender kultursensitiver Informationssysteme und deren Evaluierung fehlen bisher vollständig.

Es zeigt sich, dass innerhalb der untersuchten Beiträge auf nationaler Ebene ein homogenes Kulturverständnis im Sinne von Hofstede (1980) vorherrscht. Dies ist allerdings gerade bei so facettenreichen und sich schnell entwickelnden Ländern wie China und Indien bedenklich, da diese inzwischen eine Vielzahl von Wertemustern aufweisen (Martinsons und Ma 2009). Konsequenterweise erscheint die Nutzung einheitlicher Kulturdimensionswerte problematisch. Hier bieten sich gruppenspezifische Kulturansätze an, die auch auf nationaler Ebene Subkulturen in einem Land unterscheiden und erklären können (Abschn. 2.1). Untersucht werden sollte in diesem Zusammenhang, inwieweit divergierende Ergebnisse $\mathrm{zu}$ einzelnen Fragestellungen, die bisher vornehmlich der nationalen Kultur zugeschrieben werden, durch Sub- bzw. Gruppenkulturen erklärt werden können. Insbesondere im Marketing nutzen Forscher mit sogenannten „Ethno“Ansätzen das Potenzial von kultursensitiven Lösungen auf Gruppenebene. Fragen des Ethno-Marketing bei Endkonsumentenprodukten betreffen bspw. bestimmte Marken und Mobilfunktarife für Personen mit Migrationshintergrund in Deutschland oder spezielle Lösungen für hispanische Einwanderer in den USA. Analog könnte die Gestaltung von Informationssystemen durch eine differenzierte Kulturbetrachtung auf bestimmte Sub- bzw. Gruppenkulturen ausgerichtet werden.

Bezüglich des untersuchten Zeithorizonts wird deutlich, dass zwar oft Kon- flikte aus einem Projekt beschrieben werden (Kaye und Little 2000; Damian und Zowghi 2003; Winkler et al. 2007); longitudinale Forschungsergebnisse, die mit Datenerhebungen zu verschiedenen Zeitpunkten verbunden sind, konnten jedoch nicht identifiziert werden. Damit verbunden ist ein statisches Kulturverständnis. Veränderung von Kultur im Zeitverlauf und die korrespondierenden Steuerungsmöglichkeiten sollten daher als Forschungslücke adressiert werden. Da sich Kultur im Zeitverlauf verändert, sollte der Kultur-Fit kontinuierlich erfasst und bei der Gestaltung von Informationssystemen berücksichtigt werden. Entsprechende dynamische Ansätze und die damit einhergehenden kultursensitiven Regeln sind bisher vollkommen unerforscht.

Der Entwicklungsprozess von Informationssystemen wird im Zusammenhang mit Kultur zumeist generell untersucht. Arbeiten zu konkreten Phasen des Entwicklungsprozesses (Abschn. 4) sind eher heterogen verteilt. So konnten kaum Arbeiten in „klassischen“ Phasen der Systementwicklung wie dem Systementwurf (Tab. 1 - Themenfeld 7) oder der Implementierung (Tab. 1 Themenfeld 9) zugeordnet werden. $\mathrm{Zu}$ weiteren Phasen, wie die Verifikation und Validation (Boehm 1986) konnten gar keine Beiträge identifiziert werden. Abstrakte Gesamtaussagen helfen bei konkreten Entwicklungsvorhaben allerdings nur bedingt weiter. Aus diesem Grund sollte die zukünftige Forschung mittels geeigneter theoretischer Ansätze konkrete Handlungsabläufe untersuchen, um praktische Vorhaben zu unterstützen.

Auch das Zusammenspiel von verschiedenen Kulturarten stellt einen weiteren Forschungsbereich dar, der noch weitestgehend unerforscht ist. So wäre denkbar, dass in international besetzten Entwicklerteams die Kultur der Gruppe die nationale oder die organisationale Kultur verdrängt und dadurch bekannte kulturelle Probleme mindert. In diesem Zusammenhang sollte untersucht werden, welche Kultur wann dominiert und wie diese beeinflusst werden könnte. Dafür ist die Analyse konkreter Ursache-Wirkungsbeziehungen erforderlich, um daraus Handlungsempfehlungen und Steuerungsinstrumente abzuleiten.

Die Beiträge zwischen Gestaltungssubjekt und Gestaltungsobjekt sind zumeist allgemein gehalten und lassen 
sich nicht direkt einem bestimmten Bezugsobjekt zuordnen. Der Zusammenhang von Gestaltungssubjekt-Kultur und Gestaltungsobjekt-Kultur könnte einen tieferen Einblick in typische Konflikte geben. Ein mögliches Thema wären Kulturkonflikte, die durch die externe Systementwicklung und Systemeinführung entstehen. Zudem stellt sich auch bei der Entwicklung von Produkten für Endkunden die Frage, wie spätere Kulturunterschiede in der Nutzung bereits während der Entwicklung berücksichtigt werden können, um ein kultursensitives Gestalten $z u$ ermöglichen.

\subsection{Auf dem Weg zu einer „Theorie kultursensitiver Informationssystemgestaltung"?}

Die dritte Forschungsfrage des Beitrags bezieht sich darauf, welche Theorien im Allgemeinen und welche theoretischen Kulturmodelle im Speziellen für die Gestaltung von Informationssystemen herangezogen werden. Anhand ausgewählter Beiträge wurde dieser Aspekt bereits in Abschn. 4 erklärt. Im Folgenden werden diese Ergebnisse weiter verdichtet:

Im Rahmen der Literaturanalyse konnten Beiträge identifiziert werden, die keinen theoretischen Bezug zu Kultur beinhalten. Dabei handelt es sich oftmals um Arbeiten, die keine empirischen Daten nutzen. Hierzu gehören die Beiträge, die sich auf die Speicherung von Kultur im Sinne von kulturellem Erbe (Culture Heritage) durch Informationssysteme beziehen (Carugati et al. 2005; Garcia-Barriocanal et al. 2005; Loebbecke und Thaller 2005; Monod und Klein 2005). Die Autoren fokussieren die Gestaltungsperspektive und berücksichtigen Kultur ausschließlich als Aufgabe. Auch Davison et al. (2003) können dieser Gruppe zugeordnet werden, da ihre Ausführungen zu den E-Commerce-Möglichkeiten entlang der früheren Seidenstraße keinen kulturtheoretischen Bezug aufweisen. Die Argumentation, die diesen Beiträgen zugrunde liegt, beruht zumeist auf der Offensichtlichkeit von kulturellen Unterschieden.

Weiterhin wurden Beiträge identifiziert, die kein theoretisches Fundament erkennen lassen und auf die Identifikation von Unterschieden abzielen. Peterson und Kim (2003) beispielsweise verweisen darauf, dass Beiträge zum Software-RiskManagement primär in westlichen Ländern angesiedelt sind, und vergleichen daher die Wahrnehmung von Risikofaktoren in Japan, Korea und den USA. Darüber hinaus existieren Arbeiten, die erst bei der Erklärung der zuvor identifizierten Unterschiede theoretische Konstrukte aus der Literatur nutzen. So führen Akmanligil und Palvia (2004) Hofstede an, um Unterschiede im Outsourcing-Verhalten zu erklären.

Eine Vielzahl der identifizierten Beiträge folgt einem einheitlichen theoretischen Bezugsrahmen, um Kultur und die Gestaltung von Informationssystemen zu verbinden. Auffällig ist hierbei die starke Dominanz von Hofstedes Dimensionen (Abschn. 2.1). Diese stellen häufig den theoretischen Ausgangspunkt dar und werden auch zur Interpretation der Ergebnisse genutzt (u. a. Shanks et al. 2000; Keil et al. 2000; Leimeister et al. 2009). Alternative Konstrukte wie bspw. bei Hall und Hall (1990) konnten nur vereinzelt identifiziert werden (Choi et al. 2005; Heumann et al. 2011). Weitere Konzepte waren nicht in der betrachteten Stichprobe enthalten. Dies überrascht, da mit der GLOBE-Studie alternative Kulturdimensionen bestehen, die auf den von Hofstede basieren, dessen Ansatz jedoch durch die Unterscheidung zwischen Werten und Praktiken sowie das Hinzufügen weiterer Dimensionen erweitern.

Die Verbindung von Kulturmodellen und IS-Gestaltungsansätzen erfolgt zumeist argumentativ. Zunächst wird ein IS-Konzept mit Kulturmodellen in Verbindung gebracht. Motwani et al. (2007) beispielsweise stellen zunächst Erfolgsfaktoren von ERP-Einführungen vor und zeigen dann mögliche Zusammenhänge zu Hofstedes Kulturdimensionen auf. Anschließend beschreiben die Autoren zwei Fallstudien, in denen dieser $\mathrm{Zu}$ sammenhang anhand von empirischem Material konkretisiert wird. Kritisch ist an diesem weit verbreiteten Vorgehen, dass die Kulturdimensionen zur Erklärung genutzt werden, ohne dass eine kausale Beziehung zwischen Kultur und ISKonzept getestet wird. Ansätze, die eine tatsächliche Verschmelzung von ISKonzepten und Kulturmodellen anstreben, bestehen hingegen kaum. Lediglich Heumann et al. (2011) erfassen Kulturdimensionen in einem Fragebogen und prüfen ein ganzheitliches Modell, welches beide Themengebiete (IS und Kultur) miteinander verbindet.

Es wird deutlich, dass viele der Beiträge nicht theoriegeleitet arbeiten bzw. kaum theoretischen Nutzen für die kultursensitive Gestaltung von IS haben, da sie phänomenologisch arbeiten oder sich auf entsprechende phänomenologische Vorarbeiten beziehen. Zudem ergibt sich aus der Dominanz der Hofstede Dimensionen fast zwangsläufig, dass sich bestimmte Themenbereiche der Gestaltung gut adressieren lassen (z. B. Benutzerschnittstelle, interkulturelle Probleme in Entwicklungsteams etc.), wobei andere Bereiche (z. B. Technologie- und Architekturfragen) aber auch unzureichend erklärt werden können. Entsprechend eingeschränkt sind die Ergebnisse der Vorarbeiten für einen umfänglichen, integrierten Ansatz kultursensitiver IS-Gestaltung. Dieses Dilemma wird insbesondere in den Beiträgen von Kersten (2002) und Kersten et al. (2000, 2002) aufgezeigt, ohne jedoch konkret darzustellen, wie eine theoretische Verknüpfung zwischen der Anpassung von Kernapplikationen und nationaler Kultur erfolgen kann. Darüber hinaus dominiert, wie bereits in Abschn. 5.1 deutlich wurde, aus theoretischer Sichtweise ein vergleichsweise einfaches Verständnis von nationaler Kultur, welches statisch geprägt ist und von Homogenität innerhalb einer Nation ausgeht. Komplexere Ansätze, die Dynamik und Subkulturen erklären, werden mit Ausnahme von Walsham (2002) nicht betrachtet.

\section{Fazit}

Im vorliegenden Beitrag wurde der $\mathrm{Zu}$ sammenhang zwischen nationaler Kultur und den unterschiedlichen Bezugsobjekten der Gestaltung von Informationsund Kommunikationssystemen dargestellt. Einschränkend muss darauf hingewiesen werden, dass eine Stichprobe von Beiträgen aus der Wirtschaftsinformatikbzw. Information-Systems-Literatur durch eine strukturierte Literaturanalyse betrachtet wurde. Durch die systematische Erfassung der Beiträge wurde jedoch versucht, ein möglichst umfassendes und nachvollziehbares Bild der Forschung in diesem Bereich zu gewährleisten. Dennoch ist davon auszugehen, dass in angrenzenden Disziplinen, wie der Managementforschung oder der allgemeinen interkulturellen Vergleichsforschung weitere Beiträge bestehen, die die vorliegende Untersuchung bereichert hätten. Weiterhin ergeben sich durch den Fokus auf nationale Kultur weitreichende Einschränkungen, auf die bereits in Abschn. 2 eingegangen wurde. Obgleich Subkulturen auf nationaler 


\section{Zusammenfassung / Abstract}

Tyge-F. Kummer, Jan Marco Leimeister, Markus Bick

\section{Die Bedeutung von nationaler Kultur für die Gestaltung von Informationssystemen}

Im vorliegenden Beitrag wird anhand einer Literaturanalyse aufgezeigt, wie nationale Kultur auf die Phasen der Gestaltung von Informationssystemen einwirkt. Dabei wird einerseits der gesicherte Wissensstand aufgearbeitet, andererseits werden offene Fragestellungen aufgezeigt. Grundlage unserer Literaturanalyse bildet ein umfassender Ordnungsrahmen, der typische Dimensionen der soziotechnischen Systemgestaltung sowie zentrale Arten der Kulturforschung zueinander in Beziehung setzt. Entlang der einzelnen Dimensionen werden die bereits vorliegenden Forschungsergebnisse aus dem Bereich nationaler Kulturforschung strukturiert und den typischen Phasen der Gestaltung von Informationssystemen zugeordnet. Dabei zeigt sich, dass im Bereich Kultur und Informationssystemgestaltung häufig entweder nur das Gestaltungssubjekt oder nur das Gestaltungsobjekt adressiert wird. Arbeiten, die beide Betrachtungsebenen miteinander verbinden, existieren kaum. Weiterhin konnten kaum Publikationen zu konkreten Phasen des Entwicklungsprozesses wie dem Systementwurf, der Implementierung oder der Verifikation und Validation identifiziert werden. Aus theoretischer Perspektive fällt eine deutliche Dominanz von Hofstedes Kulturdimensionen auf. Diese können einzelne Themenbereiche der Gestaltung wie die Benutzerschnittstelle oder interkulturelle Probleme in Entwicklungsteams gut adressieren, während andere Bereiche (wie Technologie- und Architekturfragen) nur unzureichend erklärt werden können. Weiterhin wird eine überwiegend phänomenologische Ausrichtung deutlich. Die beobachteten Kulturphänomene und die damit verbundenen Interpretationen sind für konkrete Entwicklungsvorhaben nur eingeschränkt nutzbar. Der Beitrag schließt mit der Vision einer Gestaltungstheorie zur kultursensitiven Entwicklung soziotechnischer Informationssysteme, die den bestehenden Wissensstand aufnimmt und in einem strukturierten Ansatz vereint.

Schlüsselwörter: Nationale Kultur, Systemgestaltung, Sozio-technisches Systemdesign, Literaturanalyse

\section{On the Importance of National Culture for the Design of Information Systems}

In this contribution a literature review is conducted to illustrate how national culture influences phases of the design of information systems. For this purpose, we review the literature in order to identify reliable and commonly approved findings as well as still open remaining questions. Fundamentally, our literature review is a comprehensive framework that sets typical dimensions of system design as well as main types of cultural research in relation to each other. The existing research results in the area of national culture are classified along the levels of system design and attributed to typical phases of the design of information systems. It thus becomes apparent that in the domain of culture and information system design it is often only the design subject or the design object that is addressed. Contributions that connect both levels rarely exist. In our review, only a limited number of publications could be identified that covered concrete phases of the development providing system design, implementation, as well as verification and validation. From a theoretical perspective, there is an obvious dominance of Hofstede's cultural dimensions that well address single topics of the design, such as user interface and inter cultural problems in development teams. Other domains, however (e.g., technology and architecture), are inadequately explained. Further, a predominantly phenomenological focus becomes obvious. The observed cultural phenomena and the connected interpretations are usable in a limited way for concrete development initiatives. The contribution ends with the vision of a theory for the culturally sensitive design of socio technical information systems that absorbs current scientific knowledge and unites it in a structured approach.

Keywords: National culture, System design, Socio-technical system design, Literature review
Kulturebene Berücksichtigung fanden, beschränkt sich die Untersuchung auf die nationale Kulturebene. Eine Ausweitung der Analyse auf weitere Disziplinen und Kulturformen ist daher erstrebenswert.

Im Rahmen der Analyse wurden vielfältige Wirkungsbeziehungen deutlich, die anhand eines Ordnungsrahmens kategorisiert wurden. Dabei ergibt sich eine umfassende Übersicht der bestehenden Forschungslandschaft, die sich in zehn Themenbereiche untergliedern lässt (Tab. 1-A, Online-Anhang A). Entlang dieser Kategorisierung wird deutlich, dass die Ebenen des Gestaltungssubjekts und des Gestaltungsobjekts in der Forschung weitestgehend getrennt voneinander betrachtet werden. Die Wirkung von kulturellen Einflussfaktoren zwischen diesen beiden Ebenen ist jedoch von hoher Relevanz, da kulturelle Unterschiede zwischen beiden Ebenen die Entwicklung soziotechnischer Systeme beeinträchtigen. In diesem Zusammenhang können die nationale Kultur und deren Wirkungsweise genutzt werden, um die Systemgestaltung besser auf das Gestaltungsobjekt auszurichten.

Zudem fehlt eine allgemeine Gestaltungstheorie zur kultursensitiven, soziotechnischen Entwicklung von Informationssystemen. Die überwiegende Mehrheit der in der Literaturanalyse identifizierten Beiträge betrachten gleichgelagerte Phänomene aus verschiedenen Perspektiven. Eine vertiefende theoretische Fundierung dieser Ansätze hätte jedoch entscheidende Vorteile für Wissenschaft und Praxis. So hätten Forscherinnen und Forscher eine Grundlage, mittels derer sie ihre Forschung systematisieren und die Theorie falsifizieren, verallgemeinern oder erweitern könnten, während die Praxis die identifizierten Wirkungszusammenhänge auf konkrete Entwicklungsvorhaben übertragen könnte. Dies ist aufgrund der identifizierten Einzelphänomene und den mitunter stark spekulativ geprägten Deutungen dieser Beobachtungen derzeit nur bedingt möglich. Der vorgestellte Ordnungsrahmen liefert mit den Elementen der Systementwicklung unter Berücksichtigung der verschiedenen Kulturformen einen ersten Schritt zur Entwicklung eines solchen Ansatzes.

\section{Danksagung}

Die Autoren bedanken sich bei den anonymen Gutachtern und Professor Ar- 
min Heinzl für die konstruktiven Anmerkungen, die $\mathrm{zu}$ einer kontinuierlichen Verbesserung dieses Artikels beigetragen haben.

\section{Literatur}

Ågerfalk PJ, Fitzgerald B, Holmström $\mathrm{H}$, Lings B, Lundell B, Ó Conshúir E (2005) A framework for considering opportunities and threats in distributed software development. In: Proc of the international workshop on distributed software development (DiSD 2005), Austrian Computer society, S 47-61

Akmanligil M, Palvia PC (2004) Strategies for global information systems development. Inform Manage 42(1):45-59

Arnott D, Jirachiefpattana W, O'Donnell P (2007) Executive information systems development in an emerging economy. Decis Support Syst 42(4):2078-2084

Avison D, Malaurent J (2007) Impact of cultural differences: a case study of ERP introduction in China. Int J Inf Manag 27(4):368374

Becker J (1995) Strukturanalogien in Informationsmodellen - Ihre Definition, ihr Nutzen und ihr Einfluß auf die Bildung der Grundsätze ordnungsmäßiger Modellierung (GoM). In: König W (Hrsg) Strukturanalogien in Informationsmodellen - Ihre Definition, ihr Nutzen und ihr Einfluß auf die Bildung der Grundsätze ordnungsmäBiger Modellierung (GoM). Physica, Heidelberg, S 133-150

Boehm B (1986) A spiral model of software development and enhancement. Softw Eng Notes 11(4):22-41

Buhl HU, Röglinger $M$, Stöckl $S$, Braunwarth KS (2011) Wertorientierung im Prozessmanagement - Forschungslücke und Beitrag zu betriebswirtschaftlich fundierten Prozessmanagement-Entscheidungen. WIRTSCHAFTSINFORMATIK 53(3):159-169

Carugati A, Hadzilias E, Demoulin N (2005) Setting the framework for developing eGovernment services on cultural heritage. In: 13th proc European conf on information systems, Regensburg

Checkland PB (1981) Systems thinking, systems practice. Wiley, Chichester

Choe J (2004) The consideration of cultural differences in the design of information systems. Inf Manag 41(5):669-684

Choi B, Lee I, Kim J, Jeon Y (2005) A qualitative cross-national study of cultural influences on mobile data service design. In: Proc ACM conf on in human factors computing systems, Portland

Cyr D (2008) Modeling web site design across cultures: relationships to trust, satisfaction, and e-loyalty. J Manag Inf Syst 24(4):47-72

Damian DE, Zowghi D (2003) An insight into the interplay between culture, conflict and distance in globally distributed requirements negotiations. In: 26 th proc Hawaii int conf syst sci, Hawaii

Davison R, Vogel D, Harris R, Gricar J, Sorrentino M (2003) Electronic commerce on the new silk road: a conucopia of research opportunities. In: 11 th proc European conf. on information systems, Naples

Deshpande S, Richardson I, Casey V, Beecham $S$ (2010) Culture in global software development - a weakness or strength? In: 5th proc IEEE int conf on global software engineering, Princeton
Erez M, Earley PC (1993) Culture, self-identity, and work. Oxford University Press, New York

Erikson EH (1997) The life cycle completed. Norton, New York

Fettke P, Houy C, Loos P (2010) Zur Bedeutung von Gestaltungswissen für die gestaltungsorientierte Wirtschaftsinformatik. WIRTSCHAFTSINFORMATIK 52(6):339-352

Gallivan M, Srite M (2005) Information technology and culture: Identifying fragmentary and holistic perspectives of culture. Inf Organ 15(4):295-338

Garcia-Barriocanal E, Sicilia MA, Palomar D (2005) A graphical humor ontology for contemporary cultural heritage access. In 13th proc European conf on information systems, Regensburg

Hall ET, Hall MR (1990) Understanding cultura differences. Intercultural Press, Yarmouth

Hampden-Turner C, Trompenaars F (1993) The seven cultures of capitalism. Doubleday, New York

Heinrich LJ, Heinzl A, Riedl R (2011) Wirtschaftsinformatik - Einführung und Grundlegung, 4 Aufl. Oldenbourg, München

Heumann J, Wiener M, Remus U (2011) The impact of national culture on control in IS offshoring projects. In: Tagungsband Wirtschaftinformatik, Zürich

Hevner AR, March ST, Park J, Ram S (2004) Design science in information systems research. Manag Inf Syst Q 28(1):75-105

Hofstede GH (1980) Culture's consequences: international differences in work related values. Sage, Beverly Hills

Hofstede G, Hofstede GJ, Minkov M (2010) Cultures and organizations: software of the mind, 3 Aufl. McGraw-Hill, New York

Hofstede GH, Bond MH (1998) The confucius connection: from cultural roots to economic growth. Organ Dyn 16(4):4-21

Holmstrom $\mathrm{H}, \mathrm{O}^{\prime}$ Conchuir $\mathrm{E}$, Agerfalk PJ Fitzgerald B (2006) Global software development challenges: a case study on temporal, geographical and socio-cultural distance. In: Proc int conf on global software engineering, Costão do Santinho

House RJ, Javidan M (2004) Overview of GLOBE. In: House RJ, Hanges PJ, Javidan M Dorfman PW, Gupta V (Hrsg) Overview of GLOBE. Sage, Thousand Oaks, S 9-18

House RJ, Wright NS, Aditya RN (1997) Crosscultural research on organizational leadership: measurement of cultural dimensions. In: Erez PC, Earley M (Hrsg) Crosscultural research on organizational leadership: Measurement of cultural dimensions, New Lexington, San Francisco, S 571-581

Huang H, Trauth EM (2008) Cultural influences on temporal separation and coordination in globally distributed software development. In: Proc international conf on information systems, Paris

Huo YP, Randall DM (1991) Exploring subcultural differences in Hofstede's value survey: the case of the Chinese. Asia Pac J Manage 8(2):159-173

Hunter MG, Beck JE (2000) Using repertory grids to conduct cross-cultural information systems research. Inf Syst Res 11(3):93-101

Ishii K (2004) Internet use via mobile phone in Japan. Telecommun Policy 28(1):43-58

Kankanhalli A, Tan BCY, Wei K-K, Holmes MC (2004) Cross-cultural differences and information systems developer values. Decis Support Syst 38(2):183-195

Karahanna E, Evaristo JR, Srite M (2005) Levels of culture and individual behavior: an investigative perspective. J Glob Inf Manag 13(2):1-20
Kaye GR, Little S (2000) Dysfunctional development pathways of information and communication technology: cultural conflicts. J Glob Inf Manag 8(1):186-203

Keil M, Im GP, Mähring M (2007) Reporting bad news on software projects: the effects of culturally constituted views of face-saving. Inf Syst J 17(1):237-264

Keil M, Tan BCY, Wei K-K, Saarinen T, Tuuainen V, Wassenaar A (2000) A crosscultural study on escalation of commitment behavior in software projects. Manag Inf Syst Q 24(2):299-325

Kelly GA (1955) The psychology of personal constructs. Norton, New York

Kelly GA (1963) A theory of personality. Norton, New York

Kersten G (2002) Do e-business systems have culture and should they have one? In: 10th proc European conf on information systems, Gdansk

Kersten E, Matwin S, Noronha SJ, Kersten MA (2000) The software for cultures and the cultures in software. In: 8th proc European conf on information systems, Wien

Kersten GE, Kersten MA, Rakowski WM (2002) Software and culture: beyond the internationalization of the interface. J Glob Inf Manag 10(4):86-101

Kim CS, Peterson DK (2002) Cultural differences in developers' perceptions of information systems success factors: Japan vs. the United States. J Glob Inf Manag 10(2):5-13

Kroeber AL, Kluckhohn C (1952) Culture: a critical review of concepts and definitions. Peabody Museum, Cambridge

Kumar K, Bjorn-Andersen N (1990) A crosscultural comparison of IS designer values. Commun ACM 33(5):528-538

Leidner DE, Kayworth T (2006) A review of culture in information systems research: towards a theory of information technology culture conflict. Manag Inf Syst Q 30(2):357-399

Leimeister S, Leimeister JM, Knebel U, Krcmar H (2009) A cross-national comparison of perceived strategic importance of RFID for CIOs in Germany and Italy. Int J Inf Manag 29(1):37-47

Loebbecke C, Thaller M (2005) Preserving Europe's cultural heritage in the digital world. In: 13 th proc European conf on information systems, Regensburg

Marcus A, Gould EW (2000) Crosscurrents: cultural dimensions and global Web userinterface design. Interactions 7(4):32-46. doi:10.1145/345190.345238

Martinsons MG, Ma D (2009) Sub-cultural differences in information ethics across China: focus on Chinese management generation gaps. J Assoc Inf Syst 10(11):817-833

Monod E, Klein HK (2005) From ehertitage to interpretive archaeology systems (IAS): a research framework for evaluating cultural heritage communication in the digital age. In: 13th proc European conf on information systems, Regensburg

Moores TT, Gregory FH (2000) Cultural problems in applying SSM for IS development. J Glob Inf Manag 8(1):14-19

Motwani J, Akbulut AY, Gleich R, Wald A (2007) Erfolgreiche ERP-Einführungen - eine kulturvergleichende Betrachtung. HMD, Prax Wirtsch.inform 44(256):105-112

Myers M, Tan F (2002) Beyond models of national culture in information systems research. J Glob Inf Manag 10(1):24-32

oV (2008-2011) Profil der Wirtschaftsinformatik. http://wi.vhbonline.org/fileadmin/ Kommissionen/WK_WI/Profil_WI/Profil_WI _final_ds26 
Peppas SC (2001) Subculture similarities and differences: an examination of US core values. Cross Cult Manage, An Int J 8(1):59-70

Peterson DK, Kim C (2003) Perceptions on IS risks and failure types: a comparison of designers from the United States. Jpn Kor J Global Inf Manag 11(3):19-38

Piccoli G, Ives B (2005) IT-dependent strategic initiatives and sustained competitive advantage: a review and synthesis of the literature. MIS Quarterly 29(4):747-776

Rai A, Maruping LM, Venkatesh V (2009) Offshore information systems project success: the role of social embeddedness and cultural characteristics. Manag Inf Syst $Q$ 33(3):617-641

Ravesteyn P, Batenburg R (2010) Cultural differences in implementing business process management systems. In: 16th proc Americas conf on information systems, Lima

Saab DJ (2008) Anethnorelative framework for information system design. In: 14th proc Americas conf on information systems, Toronto

Sarker S, Sarker S (2009) Exploring agility in distributed information systems development teams: an interpretive study in an offshoring context. Inf Syst Res 20(3):440-461

Stahlknecht P, Hasenkamp U (2004) Einführung in die Wirtschaftsinformatik, 11 Aufl. Springer, Berlin

Schein EH (2004) Organizational culture and leadership, 3 Aufl. Jossey-Bass, San Francisco

Schreyögg G (1999) Organisation: Grundlagen moderner Organisationsgestaltung, 3 Aufl. Gabler, Wiesbaden
Schwartz SH (1992) Universals in the content and structure of values: theoretical advances and empirical tests in 20 countries. In: Zanna M (Hrsg) Universals in the content and structure of values: theoretical advances and empirical tests in 20 countries. Academic Press, New York

Shanks G, Parr A, Hu B, Corbitt B, Thanasankit T, Seddon P (2000) Differences in critical success factors in ERP systems implementation in Australia and China: a cultura analysis. In: 8th proc European conf on information systems, Wien

Straub D, Loch K, Evaristo R, Karahanna E Srite M (2002) Toward a theory-based measurement of culture. J Glob Inf Manag 10(1):13-23

Strauss W, Howe N (1991) Generations: the history of America's future. Quill William Morrow, New York, S 1584-2089

Sun H (2001) Building a culturally-competent corporate Web site: an explanatory study of cultural markers in multilingual Web design. In: Northrop MJ, Tilley S (Hrsg) Proc of the nineteenth annual ACM SIGDOC conference on computer documentation. ACM Press, New York, S 95-102

Sydow J (1988) Der soziotechnische Ansatz der Arbeits- und Organisationsgestaltung. Darstellung, Kritik, Weiterentwicklung. Frankfurt, Campus

Tajfel H (1972) Social categorization. In Moscovici S (Hrsg) Social categorization. Larousse, Paris, S 272-302

Tajfel H, Turner J (1979) An integrative theory of intergroup conflict. In: Austin WG, Worchel S (Hrsg) An integrative theory of intergroup conflict. Brooks/Cole, Monterey, S 33-47

Tan BCY, Smith HJ, Keil M, Montealegre R (2003) Reporting bad news about software projects: impact of organizational climate and information asymmetry in an individualistic and a collectivistic culture. IEEE Trans Eng Manag 50(1):64-77

Triandis HC (1982) Dimensions of cultural variations as parameters of organizational theories. Int Stud Manag Organ 12(4):139169

Walsham G (2002) Cross-cultural software production and use: a structurational analysis. Manag Inf Syst Q 26(4):359-380

Webster J, Watson RT (2002) Analyzing the past to prepare for the future: writing a literature review. Manag Inf Syst Q 26(2):1323

Wende E, Philip T (2011) Instant messenger in offshore outsourced software development projects: experiences from a case study. In: 44th proc Hawaii int conf syst sci, Hawaii

Winkler J, Dibbern J, Heinzl A (2007) Der Einfluss kultureller Unterschiede beim IT-Offshoring: Ergebnisse aus Fallstudien zu deutsch-indischen Anwendungsentwicklungsprojekten WIRTSCHAFTSINFORMATIK 49(2):95-103

Yuan M, Vogel D (2006) Cultural impact on intergroup coordination in software development in China: a qualitative analysis. In: 39th proc Hawaii int conf syst sci, Hawaii

Zahedi FM, VanPelt WV, Srite M (2006) Web documents' cultural masculinity and femininity. J Manag Inf Syst 26(1):87-128 